\title{
Vertebrate hemicentin-1
}

interacts physically and genetically with nidogen-2

\author{
Jin-Li Zhang ${ }^{1}$, Stefania Richetti ${ }^{1,{ }^{*}}$, Thomas Ramezani ${ }^{1,{ }^{*}}$, \\ Daniela Welcker ${ }^{1}$, Steffen Lütke ${ }^{2,3}$, Hans-Martin Pogoda1, \\ Julia Hatzold ${ }^{1}$, Frank Zaucke ${ }^{4}$, Douglas R. Keene ${ }^{5}$, Wilhelm Bloch ${ }^{6}$, \\ Gerhard Sengle $2,3,7,8$ and Matthias Hammerschmidtt,8,\#
}

${ }^{1}$ Institute of Zoology, Developmental Biology Unit, University of Cologne, Cologne, Germany

${ }^{2}$ Center for Biochemistry, Faculty of Medicine and University Hospital Cologne, University of Cologne, Cologne, Germany

${ }^{3}$ Department of Pediatrics and Adolescent Medicine, Faculty of Medicine and University Hospital Cologne, University of Cologne, Cologne, Germany

${ }^{4}$ Research Unit for Osteoarthritis, Department for Orthopedics, University Hospital Frankfurt, Goethe University, Frankfurt, Germany

${ }^{5}$ Micro-Imaging Center, Shriners Hospital for Children, Portland, Oregon, USA

${ }^{6}$ Institute of Cardiology and Sports Medicine, German Sport University Cologne, Cologne, Germany

${ }^{7}$ Cologne Center for Musculoskeletal Biomechanics (CCMB), University of Cologne, Cologne, Germany

${ }^{8}$ Center for Molecular Medicine Cologne (CMMC), University of Cologne, Cologne, Germany

\# author for correspondence: mhammers@uni-koeln.de

* The two authors contributed equally

Running title: hemicentin-nidogen interaction

Key words: hemicentin, nidogen, laminin, basement membrane, mouse, zebrafish 


\section{Abstract}

Hemicentins are large proteins of the extracellular matrix that belong to the fibulin family and play pivotal roles during development and homeostasis of a variety of invertebrate and vertebrate tissues. However, bona fide interaction partners of hemicentins have not been described as yet. Here, applying surface plasmon resonance spectroscopy and co-immunoprecipitation, we identify the basement membrane protein nidogen-2 (NID2) as a binding partner of mammalian hemicentin-1 (HMCN1), in line with the formerly described essential role of mouse HMCN1 for basement membrane integrity. HMCN1 binds to the same protein domain of NID2 (G2) as formerly shown for laminins, but with an approximately tenfold lower affinity and in a competitive manner. Immunofluorescence and immunogold labellings reveal that HMCN1/Hmcn1 is localized closely attached to basement membranes and in partial overlap with NID2/Nid2a in different tissues of mouse and zebrafish. Genetic knockout and antisense-mediated knockdown studies in zebrafish further show that loss of Nid2a leads to similar defects in fin fold morphogenesis as loss of Laminin- $\alpha 5$ (Lama5) or Hmcn1. Combined partial loss-of-function studies further indicate that nid2a genetically interacts with both hmon1 and lama5. Together, this suggests that despite their mutually exclusive physical binding, hemicentins, nidogens and laminins tightly cooperate and support each other during formation, maintenance and function of basement membranes to confer tissue linkage. 


\section{Introduction}

Basement membranes (BMs) are specialized thin and dense sheets of selfassembled extracellular matrix (ECM) that are present in all animal species to link or separate cells and/or tissues. Core components of BMs are laminins, collagen IV, nidogens and the heparan sulfate proteoglycans perlecan and agrin. Together, they form complex and insoluble protein meshworks around or underneath cells or tissues (1-5). In addition, BMs contain so-called matricellular proteins $(6,7)$ which, in contrast to the core components, are not required for BM assembly or architecture per se, but provide additional, often tissue-specific BM properties (3). Among them are different members of the fibulin family of ECM proteins $(8,9)$, including their largest members, the hemicentins (10-13).

The work presented here particularly focusses on interactions between mouse and zebrafish laminins, nidogens and hemicentins. Laminins are heterotrimeric proteins consisting of an $\alpha-, \beta$ - and $\gamma$-subunit joined together through a long coiled-coil domain. Five $\alpha$-, four $\beta$ - and three $\gamma$-chains have been identified in mammals; however, of the possible combinations, only 16 have been shown to exist (14). Most laminins contain the $\gamma 1$-subunit and loss of the corresponding Lamc1 gene in the mouse leads to failed BM assembly and early embryonic lethality (15). Polymerization among laminin trimers is mediated via their $\mathrm{N}$-terminal LN domains, while binding to cell surface receptors such as integrins $\alpha 3 \beta 1, \alpha 6 \beta 1, \alpha 7 \beta 1$ and $\alpha 6 \beta 4$ occurs via the C-terminal laminin-type globular (LG) domains of the a-subunit, and binding to nidogens and agrin via internal domains within the $\gamma 1$ chain (16). In contrast, binding of laminins with perlecan and collagen IV is indirect, mediated by nidogen adaptors $(3,17-19)$. 
Nidogens are glycoproteins of 150 - $200 \mathrm{kDa}$ encoded by a single gene in invertebrates, but two genes, Nid1 and Nid2, in mammals $(20,21)$, and four genes, nid1a, nid1b, nid2a and nid2b, in zebrafish (22-24). All Nidogens consist of three globular domains, $\mathrm{G} 1$ to $\mathrm{G} 3$, with $\mathrm{G} 1$ and $\mathrm{G} 2$ connected by a flexible link and $\mathrm{G} 2$ to G3 by a rod-like segment containing a series of EGF-like repeats (see Figure 1A). The G2 mediates physical binding of nidogens to collagen IV and perlecan, the G3 domain binding to laminins $(20,25-28)$. In addition, mammalian NID1 was shown to bind the matricellular protein fibulin-1 in a $\mathrm{Ca}^{2+}$-dependent manner, mediated via the C-terminal EGF-FC unit of fibulin-1, which is also present in hemicentins (8) and which consists of an array of $\mathrm{Ca}^{2+}$-binding EGF modules and a globular fibulin-type carboxy-terminal (FC) module $(29,30)$.

In mouse, concomitant loss of Nid1 and Nid2 $(31,32)$, or specific ablation of the nidogen binding site in the laminin- $\gamma 1$ chain (33), lead to wide-spread defects attributed to regional BM ruptures, such as renal agenesis and impaired lung and heart development. In contrast, Nid1 and Nid2 single mutants show very selected or no overt abnormalities (34-36), pointing to largely redundant roles of the two mouse paralogs during BM assembly and maintenance. However, even in the nematode Caenorhabditis elegans (37-39) and in the fruitfly Drosophila melanogaster (40), genetic loss of their single nidogen gene only leads to very minor defects in BM assembly, but more prominent tissue-specific defects in BMassociated functions, such as neuromuscular junctioning and longitudinal nerve positioning in C. elegans. Altogether this suggests that compared to invertebrates, vertebrate nidogens have acquired additional, more global roles for BM assembly 
(40), acting as crucial catalysts and molecular adaptors during BM de novo formation and remodeling (41-43).

Hemicentins are similarly ancient and very large members (>600 kDa) of the fibulin family. In invertebrates like the C. elegans (in which hemicentin was were initially discovered, also referred to as him-4) $(10,12,44)$ and planarian flatworms $(45,46)$, they are encoded by a single gene. In vertebrates like mammals (47-49) and zebrafish $(50,51)$, two paralogs exist, Hmcn1/hmcn1, also named fibulin-6, and Hmcn2/hmcn2. Hemicentins share a number of structural motifs (see Figure 1A): an amino-terminal von Willebrand type A (VWA) domain, followed by a long (>40) stretch of tandem immunoglobulin ( $\mathrm{lg}$ ) domains, a G2F domain combined with multiple tandem epidermal growth factor (EGF) domains and a fibulin carboxyterminal module (FC). Of note, the G2F-EGF modules combine to form a functional unit that apart from hemicentins is only found in nidogens, while the carboxyterminal EGF-FC modules form a functional unit that is only found in hemicentins and in the other members of the fibulin family (fibulin1-8) (8). For C. elegans HMCN, the EGF-FC module has been shown to be involved in protein polymerization and track formation (52), while initial functional investigations point to roles of HMCN in transient cell contacts that are required for cell migration and BM invasion $(10,12)$. More recent studies identified $\mathrm{HMCN}$ as part of an adhesion complex that connects juxtaposed double BMs to each other, thereby conferring tissue linkage $(5,53,54)$. Studies in planarians have further demonstrated that HMCN made by muscle cells is required for BM integrity, preventing mislocalization of neoblast stem cells and their descendants outside their normal compartments and pointing to essential roles of HMCN for proper BM function in tissue separation $(45,46)$. Similar roles 
have been suggested for mouse HMCN2 on satellite cells in skeletal muscle stem cell niches (55), while our own recent data reveal essential functions of mouse HMCN1 for proper BM integrity in dermal-epidermal junctions of the skin and in myotendinous junctions of the musculoskeletal system (49).

Previous zebrafish data from our laboratory suggest that hemicentins synergize with other ECM proteins to fulfill their essential functions during tissue linkage. We identified zebrafish hmcn1 mutants, which show fin blistering phenotypes similar to those of mutants in fras1 and frem2 (50), encoding BM-associated ECM proteins mutated in human Fraser syndrome (56), and similar to those of mutants in fibrillin2 (fbn2), encoding components of microfibrils mutated in human Congenital Contractural Arachnodactyly (57). In the zebrafish fin fold, such microfibrils might correspond to the so-called cross fibers that connect the two juxtaposed skin BMs and that even penetrate the BMs and contact the basal surface of epidermal cells (58), in line with the aforementioned matricellular and BM-linking roles of HMCN in invertebrates $(5,53,54)$. Genetic interaction studies with specific antisense morpholino oligonucleotides (MOs) injected at low doses further revealed a synergistic enhancement of fin defects upon combined partial loss of $h m c n 1$, fras 1 , frem 2 or fbn2 compared to partial loss of either of them alone. These data strongly suggest that $h m c n 1$, fras 1 , frem 2 and fbn2 functionally interact during zebrafish fin development in vivo to allow proper anchorage of the dermis and thereby tissue linkage (50). In addition, Hmcn1 seems to be required for proper interaction between the skin BM and the overlying epidermis, with mutants displaying compromised epidermal integrity, although less severe than in lama5 mutants 
lacking the laminin $\alpha 5$ chain or in itga3 mutants lacking its $\alpha 3$ integrin receptor subunit $(50,59)$.

However, the molecular basis of such functional interactions remained unclear. Indeed, as to now, no physical binding partners of hemicentin proteins have been reported in any species. Here we studied binding between mouse HMCN1 and different BM components, including laminins, collagen IV, NID1, NID2 and perlecan. Of those, only NID2 displayed strong binding, involving the G2F-EGF domain of HMCN1 (in contrast to the EGF-FC domain in the case of fibulin-1 binding to NID1; see above) and the G3 domain of NID2. Binding of HMCN1 to NID2 occurs in competition with laminin- $y 1$, but with kinetics that - together with the dynamics of HMCN1 and NID2 protein distributions in vivo - suggest that the HMCN1-NID2 interaction might be temporally restricted, with $\mathrm{HMCN} 1$ catalyzing early steps of $\mathrm{BM}$ assembly. Consistent with this notion, loss-of-function studies in zebrafish reveal that nid2a mutants display similar defects during fin fold morphogenesis as hmcn1 and lama5 mutants, affecting both the BM-dermal and the BM-epidermal junction. In addition, synergistic enhancement studies with combined partial loss-of-function scenarios reveal a genetic / functional interaction between Hmcn1 and Nid2a and between Nid2a and Lama5 in vivo, but not between Hmcn1 and Lama5 in vivo, which is in line with their biochemical binding properties in vitro. 


\section{Experimental Procedures}

\section{Recombinant expression and purification of mouse HMCN1, NID1, NID2 and their fragments}

The cDNAs encoding mouse HMCN1, NID1, NID2 and their fragments, the Laminin- $\gamma 1$ short arm and the Laminin- $\gamma 1$ short arm N836D mutant deficient in nidogen binding were cloned into a modified pCEP-Pu vector containing an $\mathrm{N}$ terminal BM-40 signal peptide and a C-terminal double Strepll-tag downstream of the restriction sites $(60,61,62)$. The recombinant plasmids were introduced into HEK293-EBNA cells (Invitrogen, Carlsbad, CA, USA) using FuGENE 6 transfection reagents (Roche, Basel, Switzerland). The cells were selected with puromycin $(1 \mu \mathrm{g} / \mathrm{ml})$ and recombinant proteins were purified directly from serum-containing cell culture medium. After filtration and centrifugation (30 min, 10,000× ), the cell culture supernatants were applied to a Strep-tactin column (IBA Lifesciences $\mathrm{GmbH}$, Göttingen, Germany) and eluted with $2.5 \mathrm{mM}$ desthiobiotin, $10 \mathrm{mM}$ Tris$\mathrm{HCl}, \mathrm{pH}$ 8.0. The eluted proteins were then purified by Fractogel ${ }^{\circledR}$ EMD TMAE (Merck KGaA, Darmstadt, Germany) or SP-Sepharose (GE-Healthcare, Chicago, IL, USA) ion exchange chromatography. Perlecan Ig10-15 fragment was expressed and purified in HEK293-EBNA cells as described above, perlecan Ig2-9 fragment was kindly provided by Takako Sasaki, Oita University, Japan. Collagen IV, collagen $\mathrm{V}$ and laminin-111 were purchased from Sigma-Aldrich (St. Louis, MO, USA), laminin 511 from BioLamina AB (Sundbyberg, Sweden). 


\section{Surface plasmon resonance analysis}

The binding of mouse HMCN1 fragments to nidogens and other matrix proteins was recorded on a BIAcore 3000 system (GE-Healthcare). Protein ligands in $10 \mathrm{mM}$ sodium acetate at $\mathrm{pH} 4$ were coupled to the carboxymethylated dextran layer of CM5 sensorchips which were activated with $0.05 \quad \mathrm{M}$ N-ethyl-N'-(3diethylaminopropyl)-carbodiimide and $0.05 \mathrm{M} \mathrm{N}$-hydroxysuccinimide following a manufacturer's protocol. Interaction was analyzed by recording sensograms in HBS buffer (10 mM HEPES, pH7.4, 150 mM NaCl, 2.5 mM CaCl $2,0.005 \%$ surfactant P20). Surfaces were regenerated by perfusion for 1.5 min with $4 \mathrm{M} \mathrm{MgCl}_{2}$. All measurements were corrected for non-specific interactions by subtracting a control sensogram recorded for flow cell 1. For dissociation constants $\left(K_{D}\right)$ of $<50 \mathrm{nM}$, kinetic constants ( $k_{\text {on }}$ and $k_{\text {off }}$ ) were evaluated. For $K_{D}$ values of $>50 \mathrm{nM}$, dosedependent equilibrium binding was evaluated. Per experiment, $K_{D}$ values or rate constants $k_{\text {off }} / k_{\text {on }}$ were determined for 6-9 different concentrations of the analytes. Mean values of $K_{D}$ and their standard deviation (S.D.) values were calculated from the values of at least three different experiments. S.D. values for the obtained affinities were less than $50 \%$.

\section{Co-precipitation studies}

The cDNA encoding mHmcn1 fragment G2F-EGF1-5 (H1-G2E1-5) with His 6 -tag was subcloned into pCEP4 vector containing hygromycin resistance gene. The construct was transfected into HEK293-EBNA cells containing the plasmid pCEPpu-mNidogen2-strep-tag or pCEP-pu-mNidogen2-TG3-strep-tag (both containing a puromycin resistance). The cells were selected with puromycin 
$(1 \mu \mathrm{g} / \mathrm{ml}) /$ hygromycin $(100 \mu \mathrm{g} / \mathrm{ml})$ and the confluent cells were cultured for 3 days. The conditioned medium was recovered by centrifugation, and $1 \mathrm{ml}$ of the conditioned medium was mixed with $40 \mu \mathrm{l}$ of $\mathrm{Ni}^{2+}-\mathrm{NTA}$ sepharose beads (Invitrogen) at $4^{\circ} \mathrm{C}$ for $1 \mathrm{~h}$. The beads were then washed for three times with PBS, $\mathrm{pH} 7.4$ at $4^{\circ} \mathrm{C}$, centrifuged at $500 \mathrm{rpm}$ for $5 \mathrm{~min}$, and boiled in SDS-PAGE loading buffer for $3 \mathrm{~min}$. The supernatant was separated into two parts and subjected to SDS-gel electrophoresis under reducing conditions and immunoblotting. NID2 proteins were detected by HRP-conjugated strep-tactin (IBA-GmbH), and H1G2E1-5 was detected by mouse anti-His6-tag antibody (Invitrogen) and subsequently by HRP-conjugated secondary antibody (Jackson ImmunoResearch). As a negative control, conditioned medium from cultured HEK293-EBNA cells transfected with NID2-strep or NID2-TG3-strep alone was precipitated by $\mathrm{Ni}^{2+}$-NTA sepharose beads and detected by anti-His 6 -tag antibody in parallel.

To test whether His6-tagged H1-G2E1-5 could be co-precipitated by Strep-tagged NID2 or NID2-TG3, in a reverse setup the experiments were done in the same way, but the conditioned medium was precipitated by Strep-tactin-sepharose (IBA$\mathrm{GmbH}$ ) and the conditioned medium from cultured HEK293-EBNA cells transfected with His6-tagged H1-G2E1-5 alone was taken as a negative control.

\section{Animals}

The mutant zebrafish lines $h m c n 1^{\operatorname{tg} 102}(50)$ and $\operatorname{lama}^{\mathrm{tc17}}(50)$, and the transgenic lines $\mathrm{Tg}(\mathrm{Ola} \text {.Actb:Hsa.hras-egfp) })^{v 119 T g}$ (63) and $T g(\text { krt4:tomato-CAAX) })^{\text {fr48Tg }}$ were previously described. Mutant line nid2a $a^{s a 15802}$, generated via ENU chemical 
mutagenesis, was purchased from the European Zebrafish Resource Center (EZRC, https://www.ezrc.kit.edu). It contains an A-G nonsense point mutation leading to premature termination of the Nid2a protein at aa 437 and nonsensemediated decay of the mutant transcript, most likely representing an amorph or

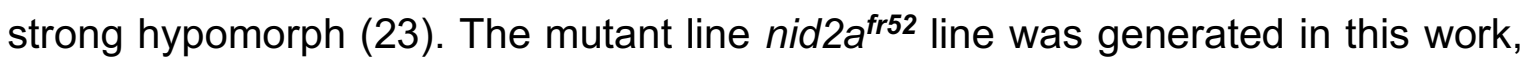
using CRISPR-Cas9 technology. The reverse strand of nid2a-exon2 from position bp73 to 92 was chosen as target site using the ZiFiT-web-tool (http://zifit.partners.org/ZiFiT/). Synthetic dsDNA of a dedicated gRNA scaffold (65) $\begin{array}{lllll}\text { was } & \text { fbtained } & \text { (Belgium): }\end{array}$ GCGTAATACGACTCACTATAGGCGATGACGGGGAAGTCGGTGTTTTAGAGCT AGAAATAGCAAGTTAAAATAAGGCTAGTCCGTTATCAACTTGAAAAAGTGGCA CCGAGTCGGTGCTTTAAACGCG-3'. This nid2a-exon2 gRNA template was cloned via TA-cloning into the $\mathrm{pCR}^{\mathrm{TM}} \|$ vector (Invitrogen). Sequence verified plasmid-DNA was linearized by Dral digest, $\mathrm{PCl}$ extracted following standard protocols and subsequently transcribed using the MAXIscript ${ }^{\mathrm{TM}} \mathrm{T7}$ Transcription Kit (Invitrogen). The obtained nid2a-exon2-gRNA was extracted using Microspin ${ }^{\mathrm{TM}} \mathrm{G}-$ 50 columns (Sigma). Cas9 mRNA was generated as reported (66). Injection mixes

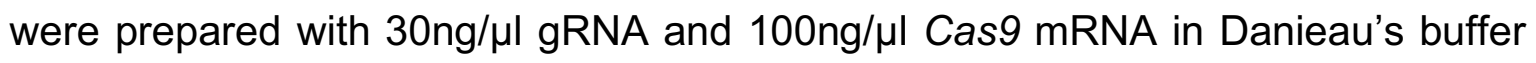
supplemented with $0.05 \%$ Phenol Red. Embryos were injected at the one-cell-stage and raised to adulthood. F1 individuals were screened for germline transmission by PCR using the primers 5'-CAGGATCTTCCCATGGAGAA-3'; 5'CTTTTGACATCCGCTTCTGC-3' followed by a T7 endonuclease assay (NEB) to detect indels. Subsequent sequencing of indel-harboring amplicons led to the identification of an allele with a 7bp (TTTTTCC) insertion in exon 2 of the nid2a gene, leading to a frame shift at amino acid residue (aa) 107 of the normally 1576 
aa protein, N-terminal of the first annotated domain (NIDO domain; $113-280$ ), and premature truncation of the protein after additional 14 amino acid residues (FFRLPRHRPL SGRH). This allele, which most likely represents a functional null (amorph), was used to establish the stable nid2 $a^{\text {fr52 }}$ line. Genotyping was performed via PCR of genomic DNA with exon 2 primers, allowing electrophoretic sizediscrimination of wild-type and mutant bands on a $3.5 \%$ agarose gel in TBE buffer.

Zebrafish and mice were kept under standard conditions, zebrafish embryos were incubated at $28^{\circ} \mathrm{C}$ in $\mathrm{E} 3$ medium $(5 \mathrm{mM} \mathrm{NaCl}, 0.17 \mathrm{mM} \mathrm{KCl}, 0.33 \mathrm{mM} \mathrm{CaCl}, 0.33 \mathrm{mM}$ $\left.\mathrm{MgSO}_{4}\right)$

Zebrafish and mouse breeding and zebrafish experiments were approved by the national animal care committees (LANUV Nordrhein-Westfalen; 40.15.097; 8402.04.2012.A251; 84-02.04.2016.A390; 84-02.04.2018.A281; City of Cologne; 576.1.36.6.G13/18 Be) and the University of Cologne (4.16.024; 4.18.013).

Zebrafish RNA and Morpholino oligonucleotide injections for loss-offunction, rescue and synergistic enhancement studies

Morpholino oligonucleotides (MOs) targeting translational start or splice sites were ordered from Gene Tools (Philomath, OR), dissolved in distilled water to $1 \mathrm{mM}$ stock solutions and kept at room temperature. For injection, aliquots were heated to $65^{\circ} \mathrm{C}$, centrifuged and supernatants were diluted in Danieau's buffer and Phenol Red (0.05\%; Sigma) (67). $2.5 \mathrm{nl}$ of MO solution was injected per embryo at the 1-4 cell stage using glass needles pulled on a Sutter needle puller and a Nanoject injection apparatus (Word Precision Instruments). MOs used and their sequences (given 5'3') were as follows: 
hmcn1-ATG: AAA ACG GCG AAG TTA TCA AGT CCA T

lama5-splice:AAC GCT TAG TTG GCA CCT TGT TGG C

nid2a -1.ATG: TTT TTG GCG AGC AGC TGG TAC ACA T

nid2a-2.ATG: CAG CTG GTA CAC ATG CAG CTC GTG A

nid2a-3.ATG: AGC ATG CGG GAC TCT GGC TTT GAA T

nid1b-ATG: CTG TAA AAC TCC ACA TAC ACG GGC T

Of the three nid2a MOs, nid2a-1.ATG gave stronger phenotypes than the two others and was used in all shown experiments. To control the efficacy of the nid2a1.ATG MO, anti-Nid2a immunoblotting and immunofluorescence studies were performed. To control its specificity, rescue experiments with co-injected mouse Nid2 mRNA were performed, which in the targeted 25 bp region contains 15 mismatches compared to the zebrafish nid2a cDNA and the morpholino sequence. To generate full-length mouse Nid2 mRNA, plasmid pUC19-mNidogen2 (68) was cut with Xbal, followed by blunting with Klenow and cutting with Xhol. The obtained fragment was cloned into the Stul and Xhol sites of the pCS2+ expression vector and the resulting plasmid was linearized with Notl and transcribed using the SP6 mMessage Machine Kit (Ambion/ThermoFisher). $2.5 \mathrm{nl}$ mNidogen-2 mRNA was injected per embryo at concentrations of $200 \mathrm{ng} / \mu \mathrm{l}$ alone or together with $1 \mathrm{ng} / \mu \mathrm{l}$ nid2a-1.ATG-MO.

To control the efficacy of the nid1b-ATG MO, a 135bp sequence of zebrafish nid $1 b$ with $81 \mathrm{bp}$ of 5 'UTR and the first 18 codons of the ORF were PCR amplified from 24hpf cDNA using the primer pair 5'GGAAGGATCCACTGCAGACCGGGGCTCCTC-3' (forward) and 5'- 
GGAAGAATTCCTGGACCGAGACCAGCAGAGC-3' (reverse). The fragment was cloned via BamHI and EcoRI in frame into expression plasmid XLT.GFPLT-CS2+ (gift from Randall Moon; Addgene plasmid \# 17098). The integrity and functionality of the construct was verified by sequencing. To obtain capped nid1b-GFP fusion mRNA for injections the plasmid was linearized with Notl and transcribed using the SP6 mMessage Machine Kit (Ambion/ThermoFisher). $2.5 \mathrm{nl}$ nid1b-GFP mRNA was injected at a concentration of $80 \mathrm{ng} / \mu \mathrm{l}$ alone or together with $1 \mathrm{ng} / \mu \mathrm{ln}$ nid $1 \mathrm{~b}-A T G$ MO. Injected embryos were assayed for GFP expression at $80 \%$ epiboly stage, using a Leica M165FC fluorescence binocular.

\section{Generation of zebrafish Hmcn1 and Nid2a antibodies}

cDNA constructs encoding amino acid residues 917 - 1352 of zebrafish Nid2a and residues 23-436 of zebrafish Hmcn1 were generated by RT-PCR on total RNA from 24 hpf embryos and cloned with 5'-terminal Nhel and 3'-terminal Xhol restriction sites. PCR products were inserted into a modified pCEP-Pu vector containing an $\mathrm{N}$-terminal BM-40 signal peptide and a C-terminal strepll-tag downstream of the restriction sites (61). Recombinant zebrafish Nid2a and Hmcn1 fragments were generated in HEK293-EBNA cells (Invitrogen) and purified as described above for the mouse proteins and used to immunize rabbits or guinea pigs (Pineda Antikörper Service, Berlin, Germany). Obtained antisera were purified by affinity chromatography on a column with purified Nid2a or Hmcn1 fragment coupled to CNBr-activated Sepharose (GE Healthcare). Specific antibodies were eluted with $3 \mathrm{M} \mathrm{KSCN}$, and the eluate was dialyzed against PBS, $\mathrm{pH} 7.4$. 


\section{Mouse and zebrafish sectioning and immunofluorescence studies}

Mouse skin tissue was fixed for $2 \mathrm{~h}$ in $4 \%$ paraformaldehyde and then frozen in optimal cutting temperature compound (OCT, Sakura,Torrance,CA) at $-80^{\circ} \mathrm{C}$. Mouse skeletal muscle tissue (m. gastrocnemius) was immediately embedded in OCT after harvesting, and frozen in liquid nitrogen-cooled isopentane. The frozen samples were cryosectioned at $8-12 \mu \mathrm{m}$ and stored at $-20^{\circ} \mathrm{C}$. Muscle sections were fixed in $100 \% \mathrm{EtOH}$ for 15 minutes. After several washes, sections were blocked with $10 \%$ sheep serum for 1 hour and then incubated different primary antibodies in blocking solution: guinea pig-anti-mHMCN1 (1653 - 2275 aa) (69), rabbit-anti-mHMCN2 (4429 - 4753 aa) (69), rabbit-anti-mNID2, rabbit-antimLaminin- $\gamma 1$ antiserum (both kindly provided by Takako Sasaki, Oita University, Japan). After overnight incubation at $4^{\circ} \mathrm{C}$ the sections were again washed with PBS and incubated with the appropriate secondary antibodies conjugated to Alexa 488 or 555 (Molecular Probes, Eugene, OR, USA) at RT for $2 \mathrm{~h}$.

Zebrafish embryos were fixed $2 \mathrm{~h}$ in $4 \%$ paraformaldehyde, washed extensively in PBS-Triton X-100 (0.5\%), blocked in blocking buffer (10\% fetal calf serum and $1 \%$ DMSO, in PBS-Triton X-100 0.5\%), and incubated at $4^{\circ} \mathrm{C}$ with the primary antibody: rabbit-anti-zfNid2a (this work), guinea pig-anti-zfHmcn1 (this work), rabbit-anti-panLaminin (Sigma-Aldrich, L9393), chicken anti-GFP (Invitrogen, A10262). The following secondary antibodies conjugated to Alexa 488, 555 and 647 (Molecular Probes, Eugene, OR, USA) were used in blocking buffer. Confocal images were obtained using a Zeiss LSM 710, 40×/1.1 W Korr LD C-Apochromat, or 20×/0.8 Plan-Apochromat objective and Zen 2.3 SP1 software. Images were processed 
using $\mathrm{Fiji} /$ ImageJ software to obtain maximum intensity projections, and for adjustment of brightness and contrast.

\section{Stimulated emission depletion (STED) microscopy}

Confocal and STED images were acquired using a gSTED microscope (Leica Microsystems) equipped with a white light laser for excitation. A $592 \mathrm{~nm}$ depletion laser was used for Alexa 488 and a $775 \mathrm{~nm}$ depletion laser for the two other dyes (Abberior STAR 580 and Abberior STAR 635P). A 100x oil immersion objective with a numerical aperture of 1.4 (Leica Microsystems) was used, and the three channels were acquired in a sequential mode. Deconvolution of the images was done using the software Huygens Essential (Scientific Volume Imaging).

\section{Zebrafish whole mount in situ hybridizations}

Embryos were fixed in $4 \%$ PFA in PBS overnight at $4^{\circ} \mathrm{C}$, and in situ hybridizations were performed as previously described (70) with RNA probes generated from linearized plasmids using the Roche digoxygenin RNA synthesis kit. For nid1a, plasmid pExpress1-zfnid1a (IMAGp998B0919858Q; NCBI-Acc.-No. EL646124) with a 843 bp nid 1a cDNA fragment was linearized with Smal and transcribed with T7 RNA polymerase; for nid1b, plasmid pAMP1-zfnid1b (IMAGp998P0611194Q; NCBI-Acc.-No. EL646124) with a 486 bp nid1b cDNA fragment was linearized with EcoRI and transcribed with SP6 RNA polymerase, for nid2a, plasmid pBluescriptzfnid2a (recloned from IRALp962C1766; NCBI-Acc.-No. CK682403) with a 490bp nid2a cDNA fragment was linearized with Sall and transcribed with T3 RNA 
polymerase, for nid2b, plasmid pCRII-zfnid2b (recloned from IMAGp998A1717616Q; NCBI-Acc.-No. DV594608) with a 778bp nid2b cDNA fragment was linearized with $K p n l$ and transcribed with T7 RNA polymerase, for lama5, plasmid pExpress1-zflama5 (IMAGp998B1314694Q, NCBI-Acc.-No. CF348092) with a 736bp lama5 cDNA fragment was linearized with EcoRI and transcribed with T7 RNA polymerase. For hmcn1, plasmid pGEMT-hmcn1 containing a $0.75 \mathrm{~kb}$ hmcn1 cDNA fragment (51) was linearized with Notl and transcribed with SP6 RNA polymerase.

\section{Mouse and zebrafish transmission and immunoelectron microscopy}

For morphological examinations via transmission electron microscopy (TEM), zebrafish embryos were fixed in $2 \%$ glutaraldehyde and $2 \%$ PFA in $0.1 \mathrm{M}$ cacodylate buffer $(\mathrm{pH} 7.4)$ for $24 \mathrm{~h}$ at $4^{\circ} \mathrm{C}$. If not immediately processed, they were then stored at $4^{\circ} \mathrm{C}$ in $0.05 \%$ glutaraldehyde in $0.1 \mathrm{M}$ cacodylate buffer $(\mathrm{pH} 7.4)$. Fixed specimens were washed several times in PBS, post-fixed with $2 \%$ osmium tetroxide in $0.1 \mathrm{M}$ PBS for $2 \mathrm{~h}$ at $4^{\circ} \mathrm{C}$, dehydrated in a graded series of ethanol, and transferred in a reverse ethanol series to EPON-araldite (Hundsman Advanced Materials, Derry, $\mathrm{NH}$ ) (for orientation and embedding. Semithin sections (350 $\mathrm{nm}$ ) and ultrathin sections $(75 \mathrm{~nm})$ were cut with a Reichert-Jung (Leica, Wetzlar, Germany) UCT microtome. Semithin sections for light-microscopy were stained with methylene blue, ultrathin sections were mounted on copper grids coated with formvar (SPIChem), contrasted in a Leica EM AX20 contrasting machine with Laurylab Ultrastain 1 (0.5\% uranyl acetate, 15 min; Laurylab, Brindas, France) and Laurylab 
Ultrastain 2 (lead citrate, 8 min), and examined with a Zeiss EM 902 A electron microscope.

Mouse skin was immuno-gold labelled using en bloc diffusion (71) of primary guinea pig anti-mouse Hmcn1 antibodies (69), followed by secondary anti-guinea pig IgG conjugated with $0.8-$ gold particles. The $0.8-\mathrm{nm}$ gold was subsequently enhanced with additional gold precipitation followed by standard fixation and embedding for transmission electron microscopy.

Zebrafish embryos were stabilized by high pressure freezing (HPF). Briefly, intact embryos were loaded into HPF planchets, with voids surrounding the tissue replaced with $20 \%$ BSA. A second planchet was placed over the first and then the sandwich was immediately frozen in a Leica EMPact 1 High Pressure Freezer (Leica Microsystems). The two halves of the planchet were then separated under liquid nitrogen to expose the tissue within the cavity. Tissues were transferred to the frozen surface of $1 \%$ glutaraldehyde with $0.1 \%$ uranyl acetate then warmed to -90C. Utilizing a Leica Automated Freeze Substitution device (AFS), temperature was maintained for $1-3$ days at $-90^{\circ} \mathrm{C}$ followed by warming at a rate of $1^{\circ} \mathrm{C} /$ hour to $-80^{\circ} \mathrm{C}$ where the tissues remained for an additional 24 hours prior to warming at a rate of $3^{\circ} \mathrm{C} /$ hour to $-50^{\circ} \mathrm{C}$. The tissues were rinsed in $100 \%$ acetone at $-50^{\circ} \mathrm{C}$, warmed at a rate of $1^{\circ} \mathrm{C}$ per hour to $-20^{\circ} \mathrm{C}$, then rinsed again in acetone at $-20^{\circ} \mathrm{C}$ for one hour. Tissues were then infiltrated in $1: 1,1: 2$, then $1: 3$ acetone:LRWhite embedding media at $-20^{\circ} \mathrm{C}$, then warmed to $0^{\circ} \mathrm{C}$ over 3 hours. The temperature within the sample chamber of the AFS was then rapidly raised to $+20^{\circ} \mathrm{C}$ and the tissues transferred to embedding molds. Polymerization in a nitrogen atmosphere was for 24 hours at $60^{\circ} \mathrm{C}$. Ultrathin sections cut from LR White embedded zebrafish 
were mounted on formvar coated nickel slot grids, dried thoroughly, then floated onto 50 ul pools of $0.15 \mathrm{M}$ Tris- $\mathrm{HCl}, \mathrm{pH} 7.4$ (Tris) for 15 minutes, quenched in $0.05 \mathrm{M}$ glycine in $0.15 \mathrm{M}$ Tris for 60 minutes, blocked in $2 \%$ non-fat dry milk with $0.5 \%$ ovalbumin and $0.5 \%$ fish gelatin for 30 minutes, rinsed in Tris, then incubated in primary antibody diluted 1:10 in Tris for 120 minutes. Grids were then rinsed in Tris for 30 minutes, then in a combination of 5 and $10 \mathrm{~nm}$ Goat colloidal gold secondary conjugate diluted 1:10 in Tris for 60 minutes. Finally, grids were rinsed in Tris for 15 minutes, then in distilled water for 15 minutes. Sections were evaluated unstained for background and specific labeling, then contrasted for 4 minutes in $4 \%$ Uranyl acetate followed by quarter-strength Reynold's lead citrate for 15 seconds (72).

\section{Statistics}

Quantitative experiments were repeated at least three times, reaching similar results. Mean values and standard deviations of all individual specimens from one representative or all independent experiments are presented, as specified in the respective figure legends. Statistical analysis was performed using Graph Pad Prism software. For comparison of multiple groups, one-way ANOVA with post-hoc Tukey's test, for comparison of two groups, an unpaired two-tailed Student's t-test, was used to determine significance and obtained p-values are mentioned in the respective figure legends. 


\section{Results}

\section{Mouse HMCN1 protein binds to nidogens}

$\mathrm{HMCN} 1$ is a large extracellular matrix protein of over $500 \mathrm{kDa}$ consisting of more than 50 domains (Figure 1A). To identify protein binding partners of HMCN1, we chose a candidate testing approach with different recombinant BM proteins. Generation of recombinant full-length HMCN1 protein failed, most likely due to its enormous size. Therefore, we instead expressed different fragments of mouse HMCN1 that altogether span $90 \%$ of the whole protein. HMCN1 fragments consisting of the VWA domain; Ig domains 8-13, 14-20, 21-26, 27-36, 33-38 or 3944; TSR domains 1-6; the G2F domain, or a combination of subsequent TSR6-G2FEGF1-2, G2F-EGF1-5 or EGF6-8-Fc domains (Figure 1A) were isolated and purified to $>95 \%$ purity. Employing surface plasmon resonance (SPR) technology, binding of such HMCN1 fragments to collagen IV, collagen V, perlecan-Ig2-9 and Ig10-15, laminin-111, laminin-511, nidogen-1 (NID1) and nidogen-2 (NID2) was tested. Of those, only the two nidogens were found to bind HMCN1 fragments G2FEGF1-5, TSR6-G2F-EGF1-2 and G2F (Figure 1 and Table 1). However, binding of the HMCN1 fragment G2F-EGF1-5 to NID2 ( $K_{D}$ of $\left.20 \mathrm{nM}\right)$ was approximately 30 times stronger than binding to NID1 $\left(K_{D}=560 \mathrm{nM}\right)$. Experiments with additional HMCN1 fragments for further binding domain dissections were therefore concentrated on HMCN1 and NID2. These additional SPR studies revealed that HMCN1 fragment G2F-EGF1-2 binds NID2 with an affinity similar to that of TSR6G2F-EGF1-2 (Table 1), indicating that the TSR6 domain does not participate in binding. Furthermore, affinities of the G2F domain $\left(K_{D}>3 \mu \mathrm{M}\right)$ and the EGF1-5 domains $\left(K_{D}=500 \mathrm{nM}\right)$ per to Nid2 were much lower than that of the G2F-EGF1-5 
fusion $\left(K_{D}=20 \mathrm{nM}\right)$. Finally, among the 5 consecutive EGF modules of the EGF15 domain, EGF2 and EGF5 appear most important, with EGF2 increasing the binding affinity to NID2 15 times (G2F-EGF1-2 compared to G2F-EGF1) and EGF5 increasing it 10 times (G2F-EGF1-5 compared to G2F-EGF1-4), respectively (Table 1). Together, this suggests that EGF2 and 5 of HMCN1 may form a composite interface with G2F to generate high affinity to NID2. Furthermore, binding of G2FEGF1-5 to NID1 and NID2 strictly depends on the presence of $\mathrm{Ca}^{2+}$ (Table 1), suggesting that at least some of the EGF modules are $\mathrm{Ca}^{2+}$-binding (73), consistent with former findings for the binding of Nidogens to the C-terminal EGF-FC unit of fibulin-1 $(29,30)$.

Nidogens themselves are multi-domain proteins consisting of NIDO, EGF, G2F, TY and LY modules that form three globular domains called G1, G2 and G3 (Figure 1A), each of which has been shown to bind to different BM proteins (20). To further dissect the binding domain of nidogens for HMCN1, we expressed G1, G1-G2, G2 and TY-G3 (TG3) domains of mouse NID1 and NID2 and tested their binding to HMCN1 fragments. As shown in Figure 1 and Table 1, the TG3 domain of NID1 and NID2 binds to HMCN1 fragments with affinities similar to those of full-length NID1 and NID2. In contrast, no binding was found between the HMCN1 fragments and the other domains of nidogens, indicating that the binding site of nidogens for HMCN1 is restricted to its TG3 domain.

The interaction of the HMCN1 G2F-EGF1-5 fragment with full-length NID2 and its TG3 domain was further confirmed by co-precipitation experiments. To this end, Strep-tagged NID2 or NID2-TG3 was co-transfected with His 6 -tagged HMCN1G2F-EGF1-5 (H1-G2E1-5) into EBNA-293 cells. The Step-tagged proteins could be 
co-precipitated with $\mathrm{His}_{6}$-tagged H1-G2E1-5 by Ni2+-NTA resin (Figure 2A,B). In a reverse experimental setup, His 6 -tagged H1-G2E1-5 could be co-precipitated with Strep-tagged Nid2 or Nid2-TG3 by Strep-tactin-Sepharose (Figure 2C,D).

\section{HMCN1 competes with laminin for binding to the G3 domain of nidogen-2}

It is known that via their $\gamma$-chain short arms, laminins can bind to the G3 domain of NID1 and NID2 (25). As the HMCN1 binding site of NID2 is also located in G3, we wondered how the three proteins interact in concert with each other, and therefore performed competitive SPR analyses. Strikingly, H1-G2E1-5 could inhibit the binding of NID2 to laminin- $\gamma 1$ short arm (LAM $\gamma 1)$ immobilized on the chip in a concentration-dependent fashion (Figure 3A). In a reverse experimental set-up, LAM $\gamma 1$ short arm inhibited the binding of Nid2 to immobilized H1-G2E1-5, whereas a N836D LAM $\gamma 1$ short arm amino acid exchange mutant deficient in nidogen binding (62) could not inhibit NID2-HMCN1 binding (Figure 3B). Of note, the affinity between the LAM $\gamma 1$ short arm and NID2 $\left(K_{D}=k_{\text {off }} / k_{o n}=2.2 n M\right)$ was approximately 10-fold higher than that between the HMCN1 fragment H1G2E1-5 and NID2 $\left(K_{D}=\right.$ $20 \mathrm{nM}$; Table 1). This is due to an approximately 35 -fold lower dissociation rate between LAM $\gamma 1$ and NID2 $\left(\mathrm{k}_{\text {off }}=1.72 \times 10^{-4} \mathrm{~s}^{-1}\right)$ compared to HMCN1 and NID2 $\left(\mathrm{k}_{\text {off }}\right.$ $=59.9 \times 10^{-4} \mathrm{~s}^{-1}$ ), which over-compensates the approximately 3.5-fold lower association rate $\left(k_{o n}=8.01 \times 10^{4} \mathrm{M}^{-1} \mathrm{~s}^{-1}\right.$ for LAM $\gamma 1$ and NID2 versus $30.3 \times 10^{4} \mathrm{M}^{-}$ ${ }^{1} \mathrm{~s}^{-1}$ for HMCN1 and NID2). Together, this indicates that laminin competes with HMCN1 for nidogen binding, adding HMCN1 as an additional player in the nidogenlaminin-integrin network that can bind NID2 more quickly, but less persistently than laminins. 


\section{Mouse and zebrafish HMCN1/Hmcn1 proteins are localized closely adjacent to basement membranes, partially overlapping with NID2/Nid2a}

As a first step to study whether HMCN1 and NID2 also interact in vivo, we performed immunofluorescence analyses to determine the spatial distribution of the two proteins in mouse and zebrafish. Former immunofluorescence studies with specific antibodies have revealed mouse HMCN1 protein in mesenchymal tissues such as the dermis of the skin and tendons of the musculoskeletal system, while its paralog HMCN2 is localized in the epithelial counterparts such as epidermis of the skin and the endomysium of skeletal muscle (49). Double immunofluorescent labeling of skin sections with HMCN1 and NID2 antibodies (Figure 4A-C) and antiHMCN1 immunogold labeling (Figure 4D,E) further showed that in particular along follicles of epidermal appendages such as whisker follicles (Figure 4A-C) and hair follicles (Figure 4D,E) dermal HMCN1 protein accumulates directly underneath (Figure 4A,E) or even within the skin BM, partially overlapping with NID2 (Figure 4C). Similarly, endomysial HMCN2 is localized closely adjacent to NID2 and LAM 1 in skeletal muscle (Figure 4F-H).

In zebrafish, hmcn1 has been shown to be expressed in distal epidermal cells of the embryonic fin folds, where it is required for proper dermal-epidermal junction formation in developing median fin folds (50). In addition, whole mount in situ hybridization at 30 hours post fertilization (hpf) revealed a thus far undescribed expression in tenocyte precursor cells (Figure 5E), which at these embryonic stages are localized lateral to the somitic muscle, extending cellular protrusions into the somitic myosepta to contribute to myotendinous junctions $(74,75)$. Consistently, 
specific antibodies raised within the frame of this work (see Experimental Procedures) revealed Hmcn1 protein both in the dermal space between the two epidermal sheets of the median fin folds (Figure $5 \mathrm{G}, \mathrm{H}$ ), closely associated with the skin BM (Figure 5M,N), as well as in the myosepta (Figure 5G-L).

Of the four nidogen genes, nid1a, nid1b, nid2a and nid2b (ZFIN Zebrafish Information Network; ZDB-GENE-050302-58, ZDB-GENE-070802-3, ZDB-GENE030827-2, ZDB-GENE-040724-143), whole mount in situ hybridization revealed that nid1b and nid2a, but not nid1a and nid2b, are co-expressed with hmcn1 and the laminin- $\alpha 5$ gene lama5 in epidermal cells of the median fin fold (Figure $5 A-F$ ). In addition, nid1a, nid1b and nid2a are expressed by somitic muscle cells (Figure 5A-D). Furthermore, co-immunofluorescence studies with specific antibodies raised against zebrafish Nid2a (see Materials and Methods) revealed partially overlapping localization of Nid2a and Hmcn1 proteins both in the tip of the median fin fold (Figure $5 \mathrm{G}, \mathrm{H}$ ) and in the myosepta (Figure $5 \mathrm{I}-\mathrm{L}$ ). In the median fin fold, colocalization was particularly evident in distal regions, where new Hmcn1 and Nid2a proteins are made, while in more proximal regions, the Hmcn1 and Nid2a domains have started to segregate (see arrows in Figure $5 \mathrm{H}$ ). Similar regions of protein colocalization next to regions of protein segregation were revealed via STED microscopy for the myosepta. Here, regions with segregated proteins showed Nid2a located within the two juxtaposed skin BMs directly adjacent to corresponding two muscle cell membranes, but Hmcn1 between these two BMs, possibly linking them (Figure $5 \mathrm{I}, \mathrm{J})$. Together, these results indicate that at least during early stages of dermal-epidermal and myotendinous junction formation, Nid2 and Hmcn1 proteins are localized in a partially overlapping manner. 


\section{Loss of zebrafish Nid2a, Lama5 or Hmcn1 cause similar defects during median fin fold development}

To address whether hemicentins and nidogens interact functionally in vivo, we carried out comparative and combined loss-of-function studies. Mouse Hmcn1 mutants only show very mild defects in dermal-epidermal and mytendinous junctions (49), while defects do not seem further enhanced upon combined loss of Hmcn1 and Hmcn2 (48). This largely dispensable nature of mouse hemicentins precludes comparative and combined hemicentin-nidogen loss of function studies, and we therefore turned to zebrafish. Here, loss-of-function mutations in both hmon1 and the laminin- $\alpha 5$ chain gene lama5 have been shown to cause compromised dermal-epidermal junction formation in the embryonic median fin fold (50). Loss of Hmcn1 primarily affects the connection of the skin BM with the underlying dermis, causing cutaneous blistering. In comparison, the linkage between the skin BM and the overlying epidermis is less affected, only leading to moderately compromised cohesion among basal keratinocytes during more advanced stages of skin development (48 hpf) (50). This is in contrast to the defects caused by loss of integral BM components as in lama5 mutants, which display compromised linkage to overlying keratinocytes, leading to severe disintegration of the epidermis $(50,59)$, as well as compromised linkage to the underlying dermis, leading to strong dermal blistering, the main phenotypic trait of $h m c n 1$ mutants (compare panels $\mathrm{M}, \mathrm{O}$ and $\mathrm{P}$ of Figure 6).

Loss-of-function studies of zebrafish nidogen genes had thus far only been reported in the context of eye development and embryonic body size determination, but not 
for median fin fold development (22-24). Using CRISPR/Cas9 technology, we generated a genetic null mutant (nid $2 a^{f r 52}$ ) in nid2a, which of the four zebrafish nidogen genes shows an expression pattern most similar to that of hmcn1, including, together with nid1b, a shared expression in the median fin fold (see above; Figure 5). In addition, we studied the formerly described mutant allele nid2a $a^{\text {sa15802 }}(23,24)$ and also knocked down nid2a in wild-type embryos with morpholino antisense oligonucleotides (MOs) to generate nid2a morphants. Mutants and morphants displayed similar phentypes: anti-Nid2a immunofluorescences studies, which yielded strong Nid2a labelling in wild-type or un-injected siblings, failed to detect Nid2a protein in nid2a $a^{\text {fr52 }}$ mutant and nid2a morphant embryos, indicating efficient knockout and knockdown (Figure 6A-D). Similar to lama5 mutants, nid2a mutants and morphants display progressively reduced sizes of the median fin folds at $30 \mathrm{hpf}, 48 \mathrm{hpf}$ and $78 \mathrm{hpf}$ (Figure 6E-L, U), indicative of a progressive collapse of that structure as a morphological consequence of epidermal disintegration. Using transgene-encoded GFP-labelling of the cell membranes of basal keratinocyte, such compromised epidermal integrity could be directly visualized, revealing increased numbers of basal keratinocyte detachments from each other in nid2a morphants and lama5 mutants already at 30 hpf (Figure 6Q-T,V). Additionally, a rounding-up of basal keratinocytes was observed in nid2a morphants and lama5 mutants, yet not in hmon 1 mutants at this early time point (Figure 6Q-T,W). These epidermal defects most likely result from compromised BM-epidermal junction formation. In addition, nid2a mutants and morphants display cutaneous blistering similar to that of $h m c n 1$ and lama5 mutants, most likely resulting from compromised BM-dermal junction formation (Figure 6E$L, U)$. This is particularly evident in transmission electron microscopy of transverse 
sections (Figure 6M-P), revealing i) a widening of the dermal space between the two epidermal sheets of the fin fold (see also Figure 7A-H), ii) the absence of dermal collagenous fibers known as actinotrichia directly underneath the cutaneous BM of nid2a morphants, hmcn1 mutants and lama5 mutants, iii) reduced BM material in nid2a and lama5 mutants, and dermally disintegrated BM material in hmcn1 mutants. Of note, this blistering / BM-dermal junction trait of nid2a morphants is less severe and less penetrant than in hmcn1 and lama5 mutants (Figure 6U). However, it is of similar magnitude to lama5 mutants when nid $1 b$, the other nidogen gene expressed in median fin fold keratinocytes, is knocked down in addition to nid2a via injection of dedicated MOs (Supplementary Figure S1), indicating that for establishing a linkage with the underlying dermis, nid2a acts in partial functional redundancy with nid1b.

Together, these data show that, despite the competitive binding of Hmcn1 versus Laminin to Nid2 in vitro (see above, Figure 3), all three seem to cooperate to contribute to BM-epidermal as well as to BM-dermal linkage in vivo.

\section{Hmcn1, Nid2a and Laminin proteins are not required for mutual stabilization}

Members of the Fraser complex, BM-associated extracellular proteins also involved in BM-dermal linkage $(50,56,76)$, have been shown to stabilize each other $(50,77)$. To investigate whether the same might be true for Hmcn1, Nid2a and Laminins, we carried out mutual immunofluorescence studies in nid2a morphants and hmcn1 or lama5 mutants, revealing altered fin fold shapes and, to some extent, altered protein distribution patterns of Nid2a or laminin. However, labeling intensities of Nid2a in hmcn 1 and lama5 mutants, and of Laminin in hmcn 1 and nid2a mutants (Figure 7A-H) were unaltered, indicating that zebrafish Hmcn1 is not required for 
Nidogen-2 and Laminin protein stabilization, consistent with its formerly shown dispensability for Fras1 protein stability (50). Furthermore, zebrafish Nid2a is not required for Laminin stability, consistent with former immunofluorescence data for laminin- $\gamma 1$ in mouse Nid2 mutants (36). Of note, ectopic pericellular Laminin was also observed around keratinocytes in the tips of the median fin folds of nid2a morphant and lama5 mutant zebrafish embryos (Figure 7F,H, arrowheads), most likely resulting from the compromised integrity of the skin BM and the epidermis observed in the TEM analyses described above (Figure 6M-W).

\section{Zebrafish Hmcn1 genetically interacts with Nid2a during embryonic median} fin morphogenesis

Injection of lower amounts of MOs allows to generate a continuous series of hypomorphic conditions. This technique is widely used in zebrafish genetics to investigate whether combined partial knockdown of two genes has synergistically enhancing effects, indicative of a functional interaction of the two gene products in vivo (see (51) for our former data on Hmcn1, Fras1 and Fibrillin-2). Upon injection of one quarter of the $\mathrm{MO}$ amount required for full knockdown and phenotypes identical to those of null mutants, nid2a, hmon1 and lama5 partial morphants displayed wild-type morphology (normal fin fold sizes and no blistering; Figure 7I,J,M). However, when combined, hmcn1 / nid2a double partial morphants as well as nid2a / lama5 double partial morphants displayed strong fin fold size reduction (resulting from compromised BM-epidermal linkage) and strong dermal blistering (resulting from compromised BM-dermal linkage), comparable to the defects of full nid2a and full lama5 morphants. In contrast hmcn1 / lama5 double partial 
morphants showed wild-type fin fold morphology (Figure $7 \mathrm{I}, \mathrm{L}, \mathrm{M}$ ), consistent with formerly published data (50). This synergistic enhancement of defects in the hmcn1 I nid2a and nid2a / lama5 combinations indicates that the corresponding partners display a strong genetic and functional interaction in vivo, consistent with the physical binding between mammalian HMCN1 and NID2 revealed here (Figures 1 and 2), and between NID2 and laminins shown elsewhere (16). Furthermore, the absence of such a genetic interaction between Hmcn1 and Lama5 is consistent with the lack of physical binding between HMCN1 fragments and LAM 1 in our surface plasmon resonance studies (Figure 1), suggesting that their cooperation to promote BM linkage occurs in a more indirect manner (see Discussion). 


\section{Discussion}

\section{HMCN1 and NID2 physically bind each other in vitro}

With a molecular weight of over $500 \mathrm{kDa}, \mathrm{HMCN} 1$ is the largest member of the fibulin family of ECM proteins, consisting of more than 50 domains (Figure 1A). Our previous studies showed that mutations in the zebrafish hmcn1 gene result in embryonic fin blistering (50), most likely due to compromised linkage between the two adjacent epidermal BMs of the embryonic median fin folds and comparable to the essential role of $\mathrm{HMCN}$ as a BM-LINKage component connecting adjacent tissues through adjoining BMs in the nematode C. elegans $(53,54)$. More recently, we have shown that loss of HMCN1 in mice results in a widening and compromised integrity of the BM in dermal-epidermal and myotendinous junctions (49), comparable to the BM defects observed upon loss of function of hemicentin in planarians (46). However, the exact alterations within the ECM network underlying such BM integrity and linkage defects in hemicentin-deficient invertebrates and vertebrates remained unclear.

The domains of HMCN1 belong to types known to be involved in the binding among ECM / BM or between ECM / BM and cell surface proteins, suggesting that HMCN1 interacts with several other proteins to fulfill its matricellular functions. Other members of the fibulin family $(8,78)$ have been shown to physically interact with multiple proteins, including cell surface receptors like integrins (79) and the epidermal growth factor receptor (EGFR) (80), other matricellular proteins like fibronectin (81), BM proteins like laminins and nidogens (29) and fibrillary proteins like fibrillins and tropoelastin $(82,83)$. In contrast, although discovered over twenty 
year ago (10), no physical binding partners of hemicentins had been reported in any animal species thus far.

Here, testing multiple BM proteins via SPR studies, we identified nidogens as binding partners of mouse HMCN1 (Figures 1,2; Table 1). Of note, however, NID2 binds Hmcn1 with an approximately 30x higher affinity than NID1. This is in striking contrast to former results obtained for fibulin-1 (FBLN1), which only binds NID1 (28$30)$, but not NID2 $(20,84)$. Furthermore, we identified the G2F-EGF unit of HMCN1 (which is absent in FBLN1) to be involved in NID2 binding, whereas NID1 - FBLN1 binding is mediated via the EGF-FC unit of FBLN1 (which is also present in $\mathrm{HMCN} 1)$. Yet, both biochemical interactions are $\mathrm{Ca}^{2+}$-dependent. $\mathrm{Ca}^{2+}$-binding of cbEGF-domains has been shown to be essential for proper folding and proteinprotein interactions (85), and our SPR data also suggest that the presence of $\mathrm{Ca}^{2+}$ is required to guarantee structural support of cbEGF-2 and cbEGF-5 for proper binding of the G2F-EGF unit of HMCN1 to NID2.

Of note, C. elegans HMCN and FBLN-1 have been shown to recruit each other to BM-associated locations (11), while zebrafish Hmcn2 and Fbln1 have been shown to play redundant roles during dermal-epidermal junction (DEJ) formation (51). Together, this suggests that despite their different direct binding partners, NID1 and NID2 may display comparable co-operations with the HMCN - FBLN1 system.

Strikingly, of all annotated proteins, apart from hemicentins, a G2F-EGF unit as identified here as HMCN1's binding domain to the G3 domain of NID2, is only present in nidogens themselves, where it mediates binding of NID1 and NID2 to collagen IV and perlecan $(20,25,27,28)$. Theoretically, this newly identified G2F G3 binding could enable several further protein-protein interactions. First, via their 
G2F-EGF units, hemicentins could also bind collagen IV and perlecan. However, both were found negative in our initial SPR screen for binding to the different mouse Hmcn1 fragments. Second, nidogens could display intermolecular or intramolecular binding between their G2 and G3 domains, thereby driving nidogen polymerization / fibril formation and/or attenuating G2-mediated binding to perlecan or collagen IV and G3-mediated binding to laminins. Along these lines, occupancy of NID2's G3 domain by HMCN1 would prevent nidogen polymerization and promote nidogen binding to perlecan or collagen IV. However, in our SPR assays, no physical binding between G2 and G3-bearing fragments of mouse nidogens was detected (JLZ and $\mathrm{MH}$, unpublished observations), making such scenarios rather unlikely.

In contrast, we have demonstrated that HMCN1 binding to NID2's G3 domain has implications to its physical interaction with laminins $(25,26)$. Thus, binding of HMCN1's G2F-EGF to NID2's G3 domain occurs in direct competition with LAM $\gamma 1$, which is present in most laminin trimers (Figure 3). Strikingly, although the overall thermodynamic affinity of NID2 to HMCN1 is approximately 10 times lower than that to $\operatorname{LAM} \gamma 1$, the kinetic association constant between NID2 to HMCN1 is approximately 3.5 times higher than that between NID2 and LAM $\gamma 1$. These complex in vitro binding properties suggest that $\mathrm{HMCN} 1$ has a more sophisticated role in the BM niche. NID2 - HMCN1 binding may be rather temporary, with HMCN1 acting as a kind of chaperone during early steps of BM assembly, catalyzing the formation of the more stable binding of NID2 to laminins to bridge the laminin and collagen IV networks in the mature BM. 


\section{HMCN1/Hmcn1 and NID2/Nid2a co-localize in vivo}

Such a temporary function of HMCN1 - NID2 binding during early steps of BM assembly would be consistent with the dynamics of HMCN1/Hmcn1 - NID2/Nid2a localization observed in mouse and zebrafish embryos, respectively. Our former analysis with chimeric embryos have revealed that during zebrafish median fin fold development (58), BM proteins are generated by epidermal cells at the tip of the evaginating fin folds. As the fin fold grows, these cells continue to move further distally while leaving BM material behind to constitute dermal-epidermal junctions (DEJs) in more basal regions of the fin folds (50). Thus, DEJs in basal regions of the fin folds should be developmentally more advanced than in distal regions. Of note, such distal / developmentally less advanced DEJs show Hmcn1 - Nid2a colocalization, while in basal / developmentally more advanced DEJs the domains the distributions have started to segregate, with Nid2a localized within the BM and Hmcn1 directly underneath it (Figure 5). The same phenomenon was observed in DEJs along the (invaginating) whisker follicles of mouse embryos, with colocalization of HMCN1 and NID2 protein at the tips of the invaginations, but largely separated distributions at the base of the invaginations, again most likely representing developmentally less and more advanced DEJs, respectively $(86,87)$ (Figure 4). Together, this suggests that the relative distributions of $\mathrm{HMCN} 1 / \mathrm{Hmcn} 1$ and NID2/Nid2a change during the time course of BM assembly, with colocalization during early phases (when it may act as a chaperone of nidogen-laminin binding, see above), but spatial segregation during later phases.

Future studies have to reveal potential binding partners of HMCN1 during such later stages, when HMCN1 is largely located underneath, rather than directly within the 
BM (Figures 4 and 5). In addition, it will be interesting to look for potential hemicentin binding partners at cell surfaces mediating their "matricellular" functions $(3,88)$ and, at least in C. elegans, involving HMCN's N-terminal VWA domain (52). Thereby, especially the unknown contribution of mammalian HMCN2 deserves further investigation. Here, we observed that HMCN2 localizes close to NID2 and LAM 1 in the endomysium of the gastrocnemius muscle of adult mice (Figure 4F-H). This suggests that HMCN2 plays a role for BM integrity in muscle tissues, consistent with a corresponding function of HMCN2 proposed for the satellite cell niche of skeletal muscle (55).

\section{Hmcn1 and Nid2a functionally co-operate in vivo}

Importantly, in addition to the physical binding in vitro and the temporary colocalization in vivo, we could also demonstrate a functional co-operation between Hmcn1 and Nid2a in zebrafish embryos in vivo. Thus, both are required for proper formation and function of both the BM-dermal and the BM-epidermal junction (Figure 6), and both display a tight genetic interaction during dermal-epidermal junction formation, each synergistically enhancing the effect of the other upon partial loss of function (Figure 7). Of note, similar dermal-epidermal junction defects are caused by loss of function of lama5 (Figure 6$)(50,59)$, encoding the alpha chain of Laminin-511, formerly known as Laminin-10, which is also crucial for mammalian skin development (89). However, in contrast to hmcn1 and nid2a and in contrast to nid2a and lama5, hmon1 does not genetically interact with lama5 (Figure 7). This indicates that despite their competitive binding to Nid2, Hmcn1 also co-operates, 
but in a more indirect manner, with laminins to promote BM assembly and functioning, in line with the aforementioned chaperone model.

It is also noteworthy that the DEJ defects caused by loss of $h m c n 1$, nid2a or lama5, although strikingly similar, are not completely identical. For the BM-dermal junction, the blistering caused by loss of nid2a is weaker than that caused by loss of $h m c n 1$ or lama5 (Figure 6U). This is largely due to a partial functional redundancy between nid2a and nid1b during the establishment of the BM-dermal (but not the BMepidermal) junction. Thus, only the combined loss of both paralogs leads to blistering defects as strong as those of $h m c n 1$ and lama5 mutants (Supplementary Figure S1), consistent with the partially redundant roles of Nid1 and Nid2 in the mouse (31,32). Also, at the ultrastructural level, mouse (49) and zebrafish Hmcn1/hmcn1 mutants display a dermal extension of BM material not apparent upon loss of nid2a or lama5 (Figure 6M-P), pointing to an additional, possibly Nid2a and Lama5-independent or even Nid2a and Lama5-antagonistic role of Hmcn1 to spatially restrict BM formation. Similarly, for the BM-epidermal junction, hmcn1 mutants develop epidermal integrity defects significantly later (48 hpf) (50) than nid2a morphants and lama5 mutants (30 hpf; Figure $6 \mathrm{~V}, \mathrm{~W}$ ), pointing to additional functions of laminins and nidogens that are at least partly independent of Hmcn1.

It is difficult to judge whether hemicentins and nidogens display similar functional co-operations in other organisms. In mouse, the phenotype of Hmcn1 mutants $(48,49)$ is much weaker than that of Nid1/2 double mutants $(31,32)$. This is consistent with the weaker phenotypes of mutants in other BM-associated matricellular proteins compared to integral BM components (7), but largely precludes functional cooperation studies in vivo. In C. elegans, loss of the single 
nidogen (37-39) or hmcn $(10,12,53,54)$ genes lead to rather dissimilar defects, pointing to crucial functions of the two that are independent of each other. However, C. elegans HMCN and fibulin-1 proteins have been shown to recruit each other (11), and fibulin-1 has been shown to recruit nidogen (90) to shared assembly sites in vivo, suggesting that also in worms, nidogens and members of hemicentin/fibulin family can functionally interact (88) and that this interaction is highly evolutionary conserved. 


\section{Acknowledgments}

We thank Heike Wessendorf, Petra Comelli, Iris Riedl-Quinkertz and Mojgan Ghilav for excellent technical assistance, and Astrid Schauss and Christian Jüngst from the CECAD Imaging Facility for their help with STED microscopy.

\section{Funding}

Funding for this study was provided by the Deutsche Forschungsgemeinschaft (DFG), Research unit FOR2722 (ID 384170921), project grants to GS and MH (ID 407168848) and FZ (ID 407168728), and by the National Institute of General Medical Sciences (GM63904). The content of the study is solely the responsibility of the authors and does not necessarily represent the official views of the National Institutes of Health.

\section{Conflict of interest}

The authors declare no conflict of interest.

\section{Author contributions}

JZ, TR, SR, DW, SL, HMP, DK, FZ, WB, GS and MH designed experiments. JZ, TR, SR, HMP, DW, SL, DK and WB generated data. JZ, TR, SR, DW, SL, HMP, JH, DK, FZ, WB, GS and MH analyzed and processed data. JZ, GS and MH wrote the manuscript. $\mathrm{MH}$ conceptualized and supervised the project. 


\section{References}

1. Yurchenco, P. D. (2011) Basement membranes: cell scaffoldings and signaling platforms. Cold Spring Harb. Perspect. Biol. 3, a004911

2. Yurchenco, P. D. (2015) Integrating Activities of Laminins that Drive Basement Membrane Assembly and Function. Curr. Top. Membr. 76, 1-30

3. Pozzi, A., Yurchenco, P. D., and lozzo, R. V. (2017) The nature and biology of basement membranes. Matrix Biol. 57-58, 1-11

4. Jayadev, R., and Sherwood, D. R. (2017) Basement membranes. Curr. Biol. 27, R207-R211

5. Pastor-Pareja, J. C. (2020) Atypical basement membranes and basement membrane diversity - what is normal anyway? J. Cell Sci. 133, jcs241794

6. Bornstein, P. (2009) Matricellular proteins: an overview. J. Cell Commun. Signal. 3, 163-165

7. Murphy-Ullrich, J. E., and Sage, E. H. (2014) Revisiting the matricellular concept. Matrix Biol. 37, 1-14

8. de Vega, S., Iwamoto, T., and Yamada, Y. (2009) Fibulins: multiple roles in matrix structures and tissue functions. Cell. Mol. Life Sci. 66, 1890-1902

9. Nakamura, T. (2018) Roles of short fibulins, a family of matricellular proteins, in lung matrix assembly and disease. Matrix Biol 73, 21-33

10. Vogel, B. E., and Hedgecock, E. M. (2001) Hemicentin, a conserved extracellular member of the immunoglobulin superfamily, organizes epithelial and other cell attachments into oriented line-shaped junctions. Development 128, 883-894

11. Muriel, J. M., Dong, C., Hutter, H., and Vogel, B. E. (2005) Fibulin-1C and Fibulin-1D splice variants have distinct functions and assemble in a hemicentin-dependent manner. Development 132, 4223-4234

12. Vogel, B. E., Muriel, J. M., Dong, C., and Xu, X. (2006) Hemicentins: what have we learned from worms? Cell Res. 16, 872-878

13. Muriel, J. M., Dong, C., and Vogel, B. E. (2012) Distinct regions within fibulin1D modulate interactions with hemicentin. Exp. Cell Res. 318, 2543-2547

14. Aumailley, M., Bruckner-Tuderman, L., Carter, W. G., Deutzmann, R., Edgar, D., Ekblom, P., Engel, J., Engvall, E., Hohenester, E., Jones, J. C., Kleinman, H. K., Marinkovich, M. P., Martin, G. R., Mayer, U., Meneguzzi, G., Miner, J. H., Miyazaki, K., Patarroyo, M., Paulsson, M., Quaranta, V., Sanes, J. R., Sasaki, T., Sekiguchi, K., Sorokin, L. M., Talts, J. F., Tryggvason, K., Uitto, J., Virtanen, I., von der Mark, K., Wewer, U. M., Yamada, Y., and Yurchenco, P. D. (2005) A simplified laminin nomenclature. Matrix Biol. 24, 326-332

15. Smyth, N., Vatansever, H. S., Murray, P., Meyer, M., Frie, C., Paulsson, M., and Edgar, D. (1999) Absence of basement membranes after targeting the LAMC1 gene results in embryonic lethality due to failure of endoderm differentiation. J. Cell Biol. 144, 151-160

16. Mayer, U., Nischt, R., Pöschl, E., Mann, K., Fukuda, K., Gerl, M., Yamada, Y., and Timpl, R. (1993) A single EGF-like motif of laminin is responsible for high affinity nidogen binding. EMBO J. 12, 1879-1885

17. Miner, J. H., and Yurchenco, P. D. (2004) Laminin functions in tissue morphogenesis. Ann. Rev. Cell Dev. Biol. 20, 255-284 
18. Yurchenco, P. D., and Patton, B. L. (2009) Developmental and pathogenic mechanisms of basement membrane assembly. Curr. Pharm. Des. 15, 1277-1294

19. Aumailley, M. (2021) Laminins and interaction partners in the architecture of the basement membrane at the dermal-epidermal junction. Exp. Dermatol. 30, 17-24

20. Ho, M. S., Böse, K., Mokkapati, S., Nischt, R., and Smyth, N. (2008) Nidogens-Extracellular matrix linker molecules. Microsc. Res. Tech. 71, 387395

21. Zhou, S., Chen, S., Pei, Y. A., and Pei, M. (2021) Nidogen: A matrix protein with potential roles in musculoskeletal tissue regeneration. Genes \& Diseases, https://doi.org/10.1016/j.gendis.2021.1003.1004

22. Zhu, P., Ma, Z., Guo, L., Zhang, W., Zhang, Q., Zhao, T., Jiang, K., Peng, J., and Chen, J. (2017) Short body length phenotype is compensated by the upregulation of nidogen family members in a deleterious nid1a mutation of zebrafish. J. Genet. Genom. 44, 553-556

23. Carrara, N., Weaver, M., Piedade, W. P., Vöcking, O., and Famulski, J. K. (2019) Temporal characterization of optic fissure basement membrane composition suggests nidogen may be an initial target of remodeling. Dev. Biol. 452, 43-54

24. Bryan, C. D., Casey, M. A., Pfeiffer, R. L., Jones, B. W., and Kwan, K. M. (2020) Optic cup morphogenesis requires neural crest-mediated basement membrane assembly. Development 147, dev18142

25. Fox, J. W., Mayer, U., Nischt, R., Aumailley, M., Reinhardt, D., Wiedemann, H., Mann, K., Timpl, R., Krieg, T., Engel, J., and et al. (1991) Recombinant nidogen consists of three globular domains and mediates binding of laminin to collagen type IV. EMBO J. ,10, 3137-3146

26. Mayer, U., Zimmermann, K., Mann, K., Reinhardt, D., Timpl, R., and Nischt, R. (1995) Binding properties and protease stability of recombinant human nidogen. Eur. J. Biochem. 227, 681-686

27. Hopf, M., Göhring, W., Ries, A., Timpl, R., and Hohenester, E. (2001) Crystal structure and mutational analysis of a perlecan-binding fragment of nidogen1. Nat. Struct. Biol. 8, 634-640

28. Ries, A., Göhring, W., Fox, J. W., Timpl, R., and Sasaki, T. (2001) Recombinant domains of mouse nidogen-1 and their binding to basement membrane proteins and monoclonal antibodies. Eur. J. Biochem. 268, 51195128

29. Sasaki, T., Kostka, G., Göhring, W., Wiedemann, H., Mann, K., Chu, M. L., and Timpl, R. (1995) Structural characterization of two variants of fibulin-1 that differ in nidogen affinity. J. Mol. Biol. 245, 241-250

30. Adam, S., Göhring, W., Wiedemann, H., Chu, M. L., Timpl, R., and Kostka, G. (1997) Binding of fibulin-1 to nidogen depends on its C-terminal globular domain and a specific array of calcium-binding epidermal growth factor-like (EG) modules. J. Mol. Biol. 272, 226-236

31. Bader, B. L., Smyth, N., Nedbal, S., Miosge, N., Baranowsky, A., Mokkapati, S., Murshed, M., and Nischt, R. (2005) Compound genetic ablation of nidogen 1 and 2 causes basement membrane defects and perinatal lethality in mice. Mol. Cell. Biol. 25, 6846-6856 
32. Böse, K., Nischt, R., Page, A., Bader, B. L., Paulsson, M., and Smyth, N. (2006) Loss of nidogen-1 and -2 results in syndactyly and changes in limb development. J. Biol. Chem. 281, 39620-39629

33. Willem, M., Miosge, N., Halfter, W., Smyth, N., Jannetti, I., Burghart, E., Timpl, R., and Mayer, U. (2002) Specific ablation of the nidogen-binding site in the laminin gamma1 chain interferes with kidney and lung development. Development 129, 2711-2722

34. Murshed, M., Smyth, N., Miosge, N., Karolat, J., Krieg, T., Paulsson, M., and Nischt, R. (2000) The absence of nidogen 1 does not affect murine basement membrane formation. Mol. Cell. Biol. 20, 7007-7012

35. Dong, L., Chen, Y., Lewis, M., Hsieh, J. C., Reing, J., Chaillet, J. R., Howell, C. Y., Melhem, M., Inoue, S., Kuszak, J. R., DeGeest, K., and Chung, A. E. (2002) Neurologic defects and selective disruption of basement membranes in mice lacking entactin-1/nidogen-1. Lab. Invest. 82, 1617-1630

36. Schymeinsky, J., Nedbal, S., Miosge, N., Pöschl, E., Rao, C., Beier, D. R., Skarnes, W. C., Timpl, R., and Bader, B. L. (2002) Gene structure and functional analysis of the mouse nidogen-2 gene: nidogen-2 is not essential for basement membrane formation in mice. Mol. Cell. Biol. 22, 6820-6830

37. Ackley, B. D., Kang, S. H., Crew, J. R., Suh, C., Jin, Y., and Kramer, J. M. (2003) The basement membrane components nidogen and type XVIII collagen regulate organization of neuromuscular junctions in Caenorhabditis elegans. J. Neurosci. 23, 3577-3587

38. Kang, S. H., and Kramer, J. M. (2000) Nidogen is nonessential and not required for normal type IV collagen localization in Caenorhabditis elegans. Mol. Biol. Cell 11, 3911-3923

39. Kim, S., and Wadsworth, W. G. (2000) Positioning of longitudinal nerves in C. elegans by nidogen. Science 288, 150-154

40. Dai, J., Estrada, B., Jacobs, S., Sánchez-Sánchez, B. J., Tang, J., Ma, M., Magadán-Corpas, P., Pastor-Pareja, J. C., and Martín-Bermudo, M. D. (2018) Dissection of Nidogen function in Drosophila reveals tissue-specific mechanisms of basement membrane assembly. PLoS Genet. 14, e1007483

41. Aumailley, M., Battaglia, C., Mayer, U., Reinhardt, D., Nischt, R., Timpl, R., and Fox, J. W. (1993) Nidogen mediates the formation of ternary complexes of basement membrane components. Kidney Int. 43, 7-12

42. Breitkreutz, D., Mirancea, N., and Nischt, R. (2009) Basement membranes in skin: unique matrix structures with diverse functions? Histochem. Cell Biol. 132, $1-10$

43. Breitkreutz, D., Koxholt, I., Thiemann, K., and Nischt, R. (2013) Skin basement membrane: the foundation of epidermal integrity--BM functions and diverse roles of bridging molecules nidogen and perlecan. Biomed Res. Int. 2013, 179784

44. Xu, X., Xu, M., Zhou, X., Jones, O. B., Moharomd, E., Pan, Y., Yan, G., Anthony, D. D., and Isaacs, W. B. (2013) Specific structure and unique function define the hemicentin. Cell Biosci. 3, 27

45. Cote, L. E., Simental, E., and Reddien, P. W. (2019) Muscle functions as a connective tissue and source of extracellular matrix in planarians. Nat. Commun. 10, 1592 
46. Lindsay-Mosher, N., Chan, A., and Pearson, B. J. (2020) Planarian EGF repeat-containing genes megf 6 and hemicentin are required to restrict the stem cell compartment. PLoS Genet. 16, e1008613

47. Xu, X., and Vogel, B. E. (2011) A secreted protein promotes cleavage furrow maturation during cytokinesis. Curr. Biol. 21, 114-119

48. Lin, M. H., Pope, B. D., 3rd, Sasaki, T., Keeley, D. P., Sherwood, D. R., and Miner, J. H. (2020) Mammalian hemicentin 1 is assembled into tracks in the extracellular matrix of multiple tissues. Dev. Dyn. 249, 775-788

49. Welcker, D., Stein, C., Feitosa, N. M., Armistead, J., Zhang, J.-L., Lütke, S., Kleinridders, A., Brüning, J. C., Eming, S. A., Sengle, G., Niehoff, A., Bloch, W., and Hammerschmidt, M. (2021) Hemicentin-1 is an essential extracellular matrix component of the dermal-epidermal and myotendinous junctions. Sci. Rep. 11, 17926

50. Carney, T. J., Feitosa, N. M., Sonntag, C., Slanchev, K., Kluger, J., Kiyozumi, D., Gebauer, J. M., Coffin Talbot, J., Kimmel, C. B., Sekiguchi, K., Wagener, R., Schwarz, H., Ingham, P. W., and Hammerschmidt, M. (2010) Genetic analysis of fin development in zebrafish identifies furin and hemicentin1 as potential novel fraser syndrome disease genes. PLoS Genet. 6, e1000907

51. Feitosa, N. M., Zhang, J., Carney, T. J., Metzger, M., Korzh, V., Bloch, W., and Hammerschmidt, M. (2012) Hemicentin 2 and Fibulin 1 are required for epidermal-dermal junction formation and fin mesenchymal cell migration during zebrafish development. Dev. Biol. 369, 235-248

52. Dong, C., Muriel, J. M., Ramirez, S., Hutter, H., Hedgecock, E. M., Breydo, L., Baskakov, I. V., and Vogel, B. E. (2006) Hemicentin assembly in the extracellular matrix is mediated by distinct structural modules. J. Biol. Chem. 281, 23606-23610

53. Morrissey, M. A., Keeley, D. P., Hagedorn, E. J., McClatchey, S. T. H., Chi, Q., Hall, D. H., and Sherwood, D. R. (2014) B-LINK: a hemicentin, plakin, and integrin-dependent adhesion system that links tissues by connecting adjacent basement membranes. Dev. Cell 31, 319-331

54. Keeley, D. P., and Sherwood, D. R. (2019) Tissue linkage through adjoining basement membranes: The long and the short term of it. Matrix Biol. 75-76, 58-71

55. Bröhl, D., Vasyutina, E., Czajkowski, M. T., Griger, J., Rassek, C., Rahn, H. P., Purfürst, B., Wende, H., and Birchmeier, C. (2012) Colonization of the satellite cell niche by skeletal muscle progenitor cells depends on Notch signals. Dev. Cell 23, 469-481

56. Smyth, I., and Scambler, P. (2005) The genetics of Fraser syndrome and the blebs mouse mutants. Hum. Mol. Genet. 14 Spec No. 2, R269-R274

57. Putnam, E. A., Zhang, H., Ramirez, F., and Milewicz, D. M. (1995) Fibrillin2 (FBN2) mutations result in the Marfan-like disorder, congenital contractural arachnodactyly. Nat. Genet. 11, 456-458

58. Dane, P. J., and Tucker, J. B. (1985) Modulation of epidermal cell shaping and extracellular matrix during caudal fin morphogenesis in the zebra fish Brachydanio rerio. J. Embryol. Exp. Morphol. 87, 145-161

59. Webb, A. E., Sanderford, J., Frank, D., Talbot, W. S., Driever, W., and Kimelman, D. (2007) Laminin alpha5 is essential for the formation of the zebrafish fins. Dev. Biol. 311, 369-382 
60. Otten, C., Hansen, U., Talke, A., Wagener, R., Paulsson, M., and Zaucke, F. (2010) A matrilin-3 mutation associated with osteoarthritis does not affect collagen affinity but promotes the formation of wider cartilage collagen fibrils. Human Mutat. 31, 254-263

61. Maertens, B., Hopkins, D., Franzke, C. W., Keene, D. R., BrucknerTuderman, L., Greenspan, D. S., and Koch, M. (2007) Cleavage and oligomerization of gliomedin, a transmembrane collagen required for node of ranvier formation. J. Biol. Chem. 282, 10647-10659

62. Lössl, P., Kölbel, K., Tänzler, D., Nannemann, D., Ihling, C. H., Keller, M. V., Schneider, M., Zaucke, F., Meiler, J., and Sinz, A. (2014) Analysis of nidogen-1/laminin $\mathrm{y} 1$ interaction by cross-linking, mass spectrometry, and computational modeling reveals multiple binding modes. PloS One 9, e112886

63. Cooper, M. S., Szeto, D. P., Sommers-Herivel, G., Topczewski, J., SolnicaKrezel, L., Kang, H. C., Johnson, I., and Kimelman, D. (2005) Visualizing morphogenesis in transgenic zebrafish embryos using BODIPY TR methyl ester dye as a vital counterstain for GFP. Dev. Dyn. 232, 359-368

64. Teixeira Rosa, J., Oralová, V., Larionova, D., Eisenhoffer, G. T., Eckhard Witten, P., and Huysseune, A. (2019) Periderm invasion contributes to epithelial formation in the teleost pharynx. Sci. Rep. 9, 10082

65. Mali, P., Yang, L., Esvelt, K. M., Aach, J., Guell, M., DiCarlo, J. E., Norville, J. E., and Church, G. M. (2013) RNA-guided human genome engineering via Cas9. Science 339, 823-826

66. Hatzold, J., Wessendorf, H., Pogoda, H. M., Bloch, W., and Hammerschmidt, M. (2021) The Kunitz-type serine protease inhibitor Spint2 is required for cellular cohesion, coordinated cell migration and cell survival during zebrafish hatching gland development. Dev. Biol. 476, 148-170

67. Nasevicius, A., and Ekker, S. C. (2000) Effective targeted gene 'knockdown' in zebrafish. Nat. Genet. 26, 216-220

68. Salmivirta, K., Talts, J. F., Olsson, M., Sasaki, T., Timpl, R., and Ekblom, P. (2002) Binding of mouse nidogen-2 to basement membrane components and cells and its expression in embryonic and adult tissues suggest complementary functions of the two nidogens. Exp. Cell Res. 279, 188-201

69. Chowdhury, A., Herzog, C., Hasselbach, L., Khouzani, H. L., Zhang, J., Hammerschmidt, M., Rudat, C., Kispert, A., Gaestel, M., Menon, M. B., Tudorache, I., Hilfiker-Kleiner, D., Mühlfeld, C., Schmitto, J. D., Müller, M., and Theilmeier, G. (2014) Expression of fibulin-6 in failing hearts and its role for cardiac fibroblast migration. Cardiovasc. Res. 103, 509-520

70. Hammerschmidt, M., Pelegri, F., Mullins, M. C., Kane, D. A., van Eeden, F. J., Granato, M., Brand, M., Furutani-Seiki, M., Haffter, P., Heisenberg, C. P., Jiang, Y. J., Kelsh, R. N., Odenthal, J., Warga, R. M., and Nüsslein-Volhard, C. (1996) dino and mercedes, two genes regulating dorsal development in the zebrafish embryo. Development 123, 95-102

71. Sakai, L. Y., and Keene, D. R. (1994) Fibrillin: monomers and microfibrils. Methods Enzymol. 245, 29-52

72. Keene, D. R., and Tufa, S. F. (2020) Connective Tissue Ultrastructure: A Direct Comparison between Conventional Specimen Preparation and HighPressure Freezing/Freeze-Substitution. Anat Rec (Hoboken) 303, 15141526 
73. Downing, A. K., Knott, V., Werner, J. M., Cardy, C. M., Campbell, I. D., and Handford, P. A. (1996) Solution structure of a pair of calcium-binding epidermal growth factor-like domains: implications for the Marfan syndrome and other genetic disorders. Cell 85, 597-605

74. Subramanian, A., and Schilling, T. F. (2014) Thrombospondin-4 controls matrix assembly during development and repair of myotendinous junctions. eLife 3, e02372

75. Subramanian, A., Kanzaki, L. F., Galloway, J. L., and Schilling, T. F. (2018) Mechanical force regulates tendon extracellular matrix organization and tenocyte morphogenesis through TGFbeta signaling. eLife 7, e38069

76. Short, K., Wiradjaja, F., and Smyth, I. (2007) Let's stick together: the role of the Fras 1 and Frem proteins in epidermal adhesion. IUBMB Life 59, 427-435

77. Kiyozumi, D., Sugimoto, N., and Sekiguchi, K. (2006) Breakdown of the reciprocal stabilization of QBRICK/Frem1, Fras1, and Frem2 at the basement membrane provokes Fraser syndrome-like defects. Proc. Natl. Acad.Sci. U.S.A. 103, 11981-11986

78. Timpl, R., Sasaki, T., Kostka, G., and Chu, M. L. (2003) Fibulins: a versatile family of extracellular matrix proteins. Nat. Rev. Mol. Cell Biol. 4, 479-489

79. Lomas, A. C., Mellody, K. T., Freeman, L. J., Bax, D. V., Shuttleworth, C. A., and Kielty, C. M. (2007) Fibulin-5 binds human smooth-muscle cells through alpha5beta1 and alpha4beta1 integrins, but does not support receptor activation. Biochem. J. 405, 417-428

80. Harikrishnan, K., Joshi, O., Madangirikar, S., and Balasubramanian, N. (2020) Cell Derived Matrix Fibulin-1 Associates With Epidermal Growth Factor Receptor to Inhibit Its Activation, Localization and Function in Lung Cancer Calu-1 Cells. Front. Cell Dev. Biol. 8, 522

81. Balbona, K., Tran, H., Godyna, S., Ingham, K. C., Strickland, D. K., and Argraves, W. S. (1992) Fibulin binds to itself and to the carboxyl-terminal heparin-binding region of fibronectin. J. Biol. Chem. 267, 20120-20125

82. Sasaki, T., Göhring, W., Miosge, N., Abrams, W. R., Rosenbloom, J., and Timpl, R. (1999) Tropoelastin binding to fibulins, nidogen-2 and other extracellular matrix proteins. FEBS Lett. 460, 280-284

83. Thomson, J., Singh, M., Eckersley, A., Cain, S. A., Sherratt, M. J., and Baldock, C. (2019) Fibrillin microfibrils and elastic fibre proteins: Functional interactions and extracellular regulation of growth factors. Sem. Cell Dev. Biol. 89, 109-117

84. Kohfeldt, E., Sasaki, T., Göhring, W., and Timpl, R. (1998) Nidogen-2: a new basement membrane protein with diverse binding properties. J. Mol. Biol. 282, 99-109

85. Rao, Z., Handford, P., Mayhew, M., Knott, V., Brownlee, G. G., and Stuart, D. (1995) The structure of a $\mathrm{Ca}(2+)$-binding epidermal growth factor-like domain: its role in protein-protein interactions. Cell 82, 131-141

86. Millar, S. E. (2002) Molecular mechanisms regulating hair follicle development. J. Invest. Dermatol. 118, 216-225

87. Saxena, N., Mok, K. W., and Rendl, M. (2019) An updated classification of hair follicle morphogenesis. Exp. Dermatol. 28, 332-344

88. Clay, M. R., and Sherwood, D. R. (2015) Basement Membranes in the Worm: A Dynamic Scaffolding that Instructs Cellular Behaviors and Shapes Tissues. Curr. Top. Membr. 76, 337-371 
89. Li, J., Tzu, J., Chen, Y., Zhang, Y. P., Nguyen, N. T., Gao, J., Bradley, M., Keene, D. R., Oro, A. E., Miner, J. H., and Marinkovich, M. P. (2003) Laminin10 is crucial for hair morphogenesis. EMBO J. 22, 2400-2410

90. Kubota, Y., Ohkura, K., Tamai, K. K., Nagata, K., and Nishiwaki, K. (2008) MIG-17/ADAMTS controls cell migration by recruiting nidogen to the basement membrane in C. elegans. Proc. Natl. Acad. Sci. U.S.A. 105, 20804-20809 


\section{Tables}

Table 1. Dissociation constants ( $K_{D}$ values) between mouse HMCN1 fragments and full-length or G3 domains of mouse NID1 and NID2

\begin{tabular}{lcccc}
\hline HMCN1 fragments & NID2 & NID2-TG3 & NID1 & NID1-TG3 \\
\hline TSR6-G2F-EGF1-2 & $160 \mathrm{nM}$ & $176 \mathrm{nM}$ & -- & -- \\
G2F & $>3 \mu \mathrm{M}$ & $>3 \mu \mathrm{M}$ & -- & -- \\
G2F-EGF1 & $>3 \mu \mathrm{M}$ & $>3 \mu \mathrm{M}$ & -- & -- \\
G2F-EGF1-2 & $200 \mathrm{nM}$ & $185 \mathrm{nM}$ & -- & -- \\
G2F-EGF1-3 & $210 \mathrm{nM}$ & $170 \mathrm{nM}$ & -- & -- \\
G2F-EGF1-4 & $193 \mathrm{nM}$ & $180 \mathrm{nM}$ & -- & -- \\
G2F-EGF1-5 & $20 \mathrm{nM}$ & $30 \mathrm{nM}$ & $560 \mathrm{nM}$ & $585 \mathrm{nM}$ \\
G2F-EGF1-5 (no Ca $\left.{ }^{2+}\right)$ & $\mathrm{ND}$ & -- & $\mathrm{ND}$ & -- \\
EGF1-5 & $500 \mathrm{nM}$ & $620 \mathrm{nM}$ & -- & -- \\
\hline
\end{tabular}

--: Not determined; ND: not detectable 


\section{Figures}

\section{A HMCN1} SI VWA

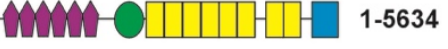

TSR G2F EGF Fc
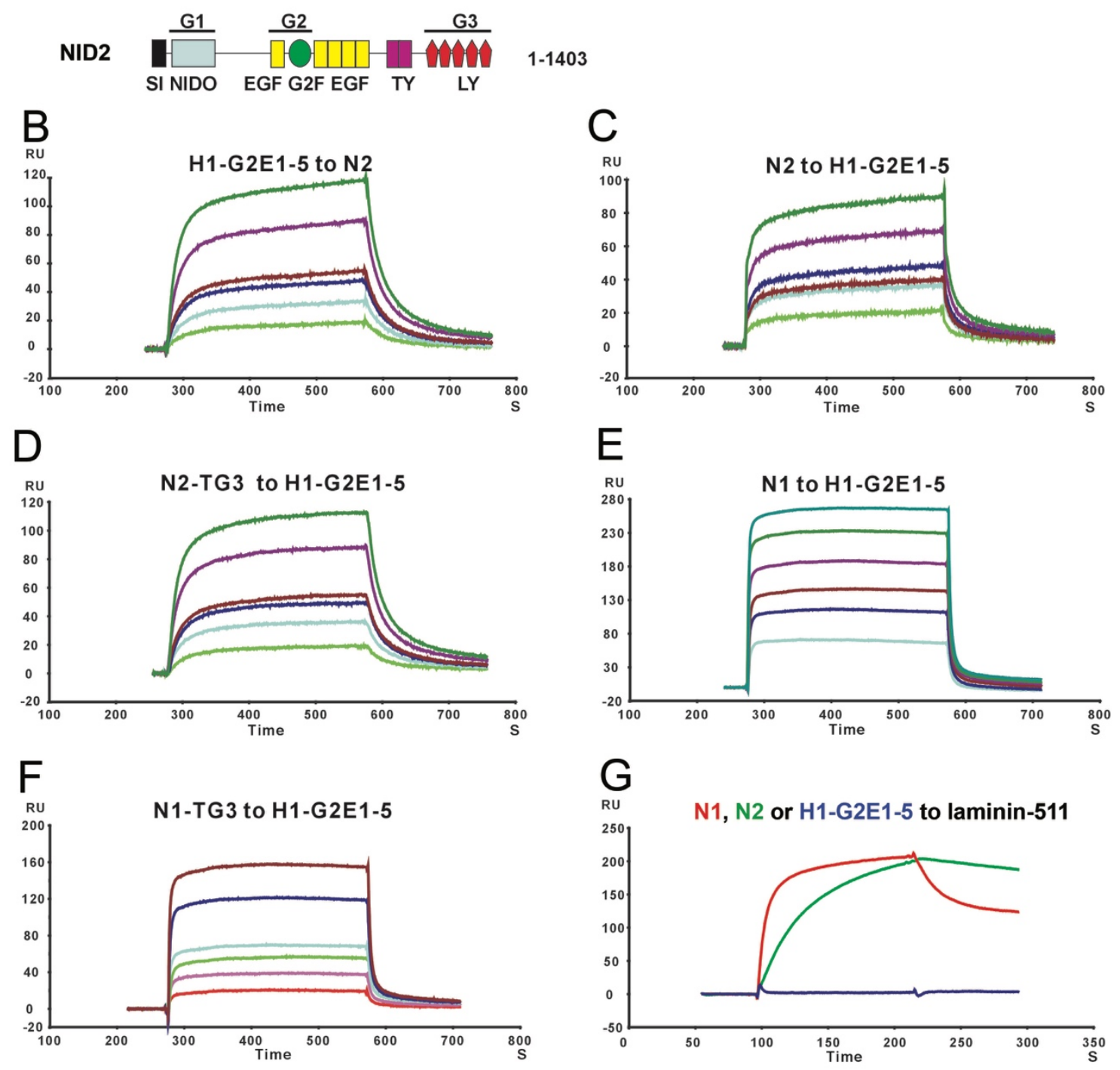

Figure 1. Surface plasmon resonance analysis of NID2 - HMCN1 binding.

A. Schematic diagram of mouse HMCN1 and NID2 proteins. Indicated modules are signal sequence (SI), von Willebrand type A (VWA), immunoglobulin (Ig), thrombospondin (TSR), nidogen G2F (G2F), epidermal growth factor (EGF), fibulin type carboxy-terminal (FCs), Nidogen-like (NIDO), thyroglobulin type-1(TY); and 
low-density lipoprotein receptor (LY) modules. The globular domains of Nidogens are indicated as G1, G2 and G3. B-F: Sensograms showing the binding of 10 (bottom curve), 20, 30, 40, 80, 120 (top curve) nM HMCN1 H1-G2E1-5 fragment to immobilized full-length NID2 (N2) (B), and the following proteins binding to immobilized HMCN1 H1-G2E1-5 fragment: 3 (bottom), 6, 9 ,12, 24, 36 (top) nM NID2 (C); 10 (bottom), 20, 30, 40, 80, 120 (top) nM NID2-TG3 domain (D); 100 (bottom), 200, 300, 400, 600, 800 (top) nM NID1 (N1) (E); 100 (bottom), 200, 300, 400, 800, 1200 (top) nM NID1-TG3 (F). G: NID1 (red) and NID2 (green), but not the HMCN1 fragment H1-G2E1-5 (blue), bind to immobilized laminin-511. Abbreviation: RU, resonance unit. 


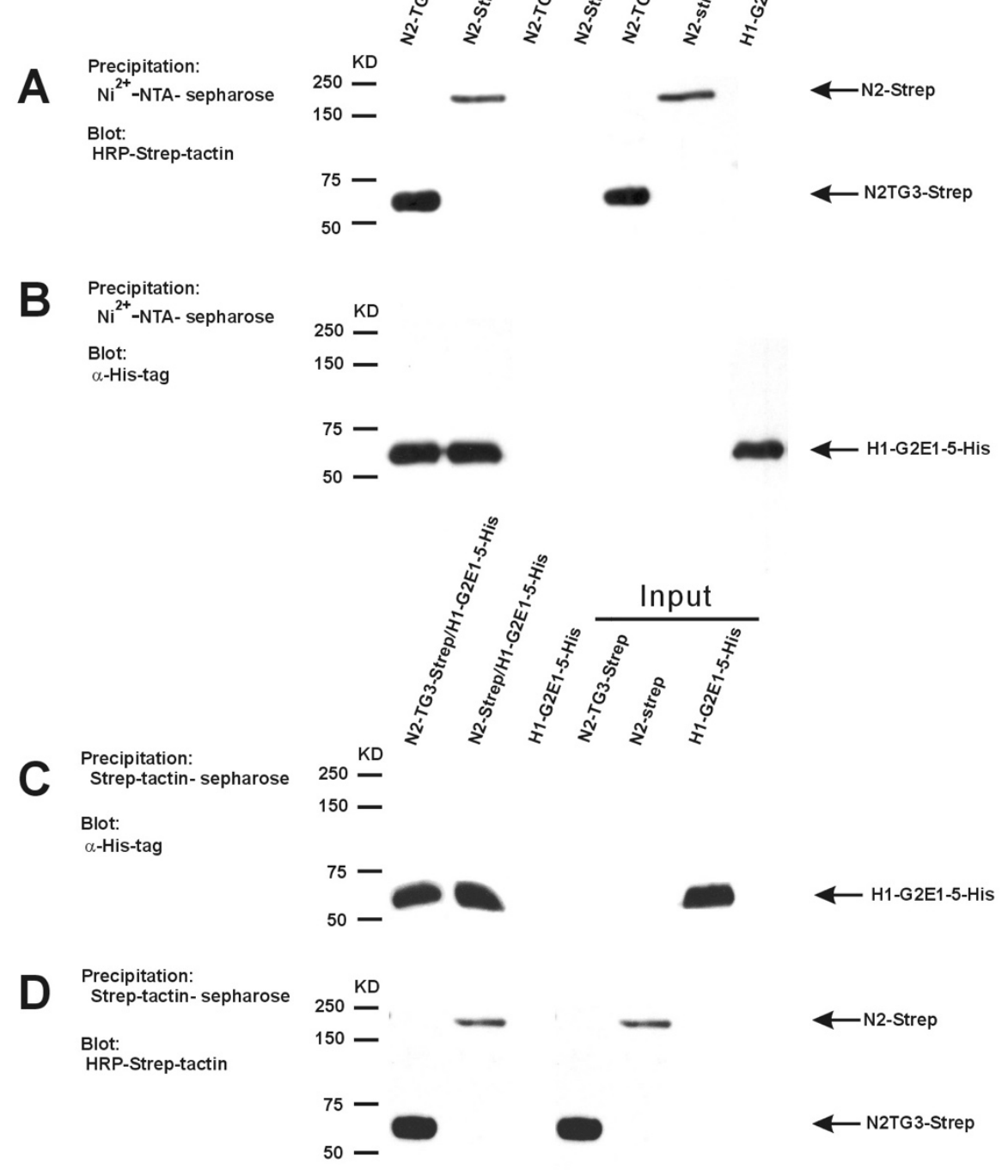

Figure 2. Analysis of NID2 - HMCN1 binding by co-precipitation.

$(A, B)$ Conditioned medium of cultured HEK293-EBNA cells transfected with NID2Strep/HMCN1-G2E1-5-His6 or NID2-TG3-Strep/HMCN1-G2E1-5-His 6 was precipitated by $\mathrm{Ni}^{2+}-\mathrm{NTA}$ sepharose and subjected to immunoblotting to detect coprecipitated Strep-tagged proteins with HRP-Strep-tactin (A) or, as control of the precipitation, $\mathrm{His}_{6}$-tagged proteins with anti-His ${ }_{6}$-tag antibodies (B) (lanes 1 and 2). As negative control, conditioned medium from cultured HEK293-EBNA cells 
transfected with NID2-strep or NID2-TG3-strep alone was precipitated by $\mathrm{Ni}^{2+}$-NTA sepharose and detected by immunoblotting (lanes 3 and 4).

$(C, D)$ For the reverse co-precipitation, conditioned medium of cultured HEK293EBNA cells transfected with NID2-Strep/ HMCN1-G2E1-5-His or N2-TG3-Strep/ HMCN1-G2E1-5-His was precipitated by Strep-tactin-sepharose and subjected to immunoblotting to detect co-precipitated $\mathrm{His}_{6}$-tagged proteins with anti-His 6 -tag antibodies $(\mathrm{C})$ or, as control of the precipitation, Strep-tagged proteins with HRPStrep-tactin (D) (lanes 1 and 2). As negative control, conditioned medium from cultured HEK293-EBNA cells transfected with HMCN1-G2E1-5-His6 alone was precipitated Strep-tactin-sepharose and detected by immunoblotting (lane 3).

Lanes 5-7 of $(A-B)$ and 4-6 of $(C, D)$ are direct loadings of the indicated proteins (50\% of input). 

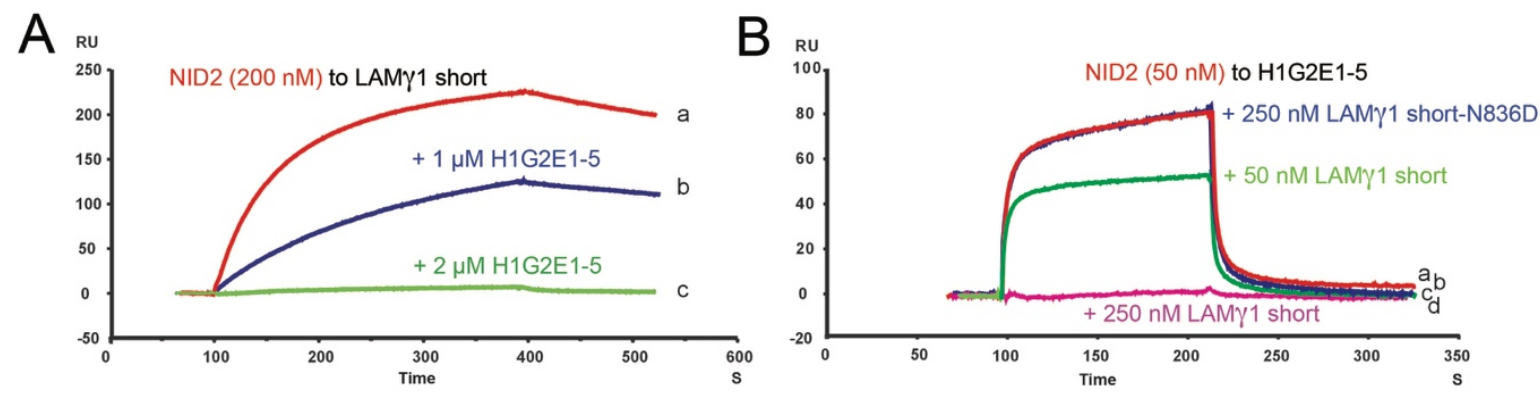

Figure 3. Surface plasmon resonance analysis of competition between HMCN1 and LAM 1 for NID2 binding.

LAM 1 short arm (A) or HMCN1 fragment HMCN1-G2E1-5 (B) were immobilized on the chip surface, and the chip was perfused with 200 nM NID2 (A, a), 200 nM NID2 plus $1 \mu \mathrm{M}$ HMCN1-G2E1-5 (A, b), 200 nM NID2 plus $2 \mu \mathrm{M}$ HMCN1-G2E1-5 (A, c), 50 nM NID2 (B, a, red), 50 nM NID2 plus 250 nM LAM 1 mutant deficient in nidogen binding mutant (N836D; 62) (B, b, blue), 50 nM NID2 plus 50 nM LAM 1 short arm (B, c, green), or 50 nM NID2 plus 250 nM LAM 1 short arm (B,d, magenta), respectively. 

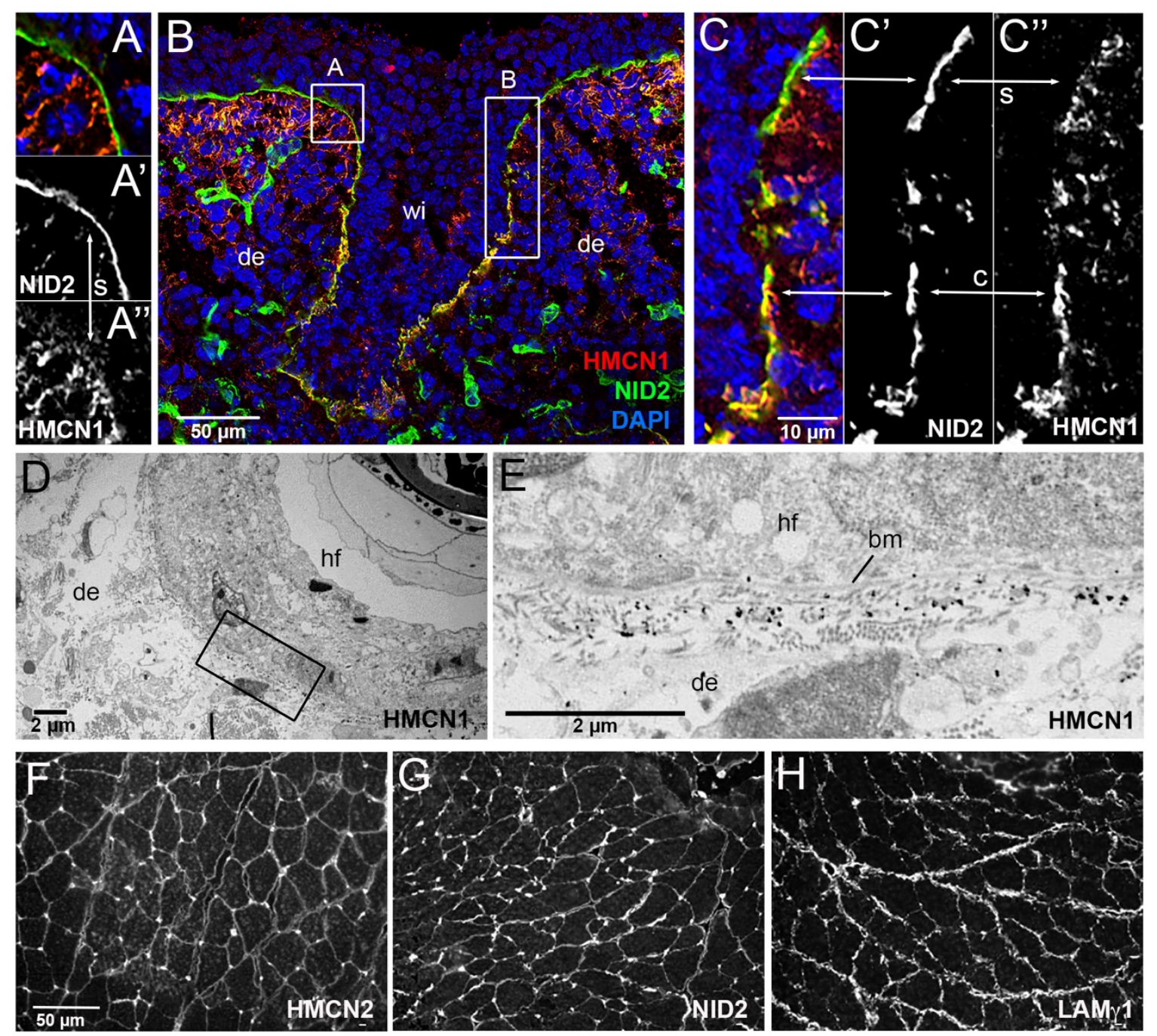

Figure 4. HMCN1 and NID2 proteins display partially overlapping localization

\section{at basement membranes of mouse embryos}

(A-C) Immunofluorescence analysis of skin at the level of the whisker follicle of embryo at E14, using antibodies directed against HMCN1 (red) and NID2 (green); the section was counterstained with DAPI to label nuclei (blue). Merged $(A, B, C)$ as well as single NID2 (A',C') and single HMCN1 (A", C") channels are shown. $(A, C)$ show magnifications of regions boxed in B. In apical regions of the whisker follicle, dermal HMCN1 protein has accumulated underneath the BM, largely, but not completely, separated from NID2 located within the BM (A,A',A",C,C',C"; indicated 
by arrows labelled with s). In contrast, in deeper regions of the whisker follicle, HMCN1 is largely located within the BM, co-localizing with NID2 (insets C,C",C"; indicated by arrows labelled with c). (D) Overview of ultrastructural immunolocalization of HMCN1 protein on skin sections of P14 mouse. (E) Higher magnification reveals clusters of HMCN1 signals underneath the BM. (F-H) Consecutive transverse sections of musculus gastrocnemius from wild-type adult mouse immunofluorescently labelled with antibodies against HMCN2 (F), NID2 (G) and LAM $1(\mathrm{H})$. HMCN2, NID2 and LAM 1 display similar distributions along endomysial BMs of muscle fibers. Abbreviations: bm, basement membrane; c, colocalized; de, dermis; hf, hair follicle; s, separated; wi, whisker. 


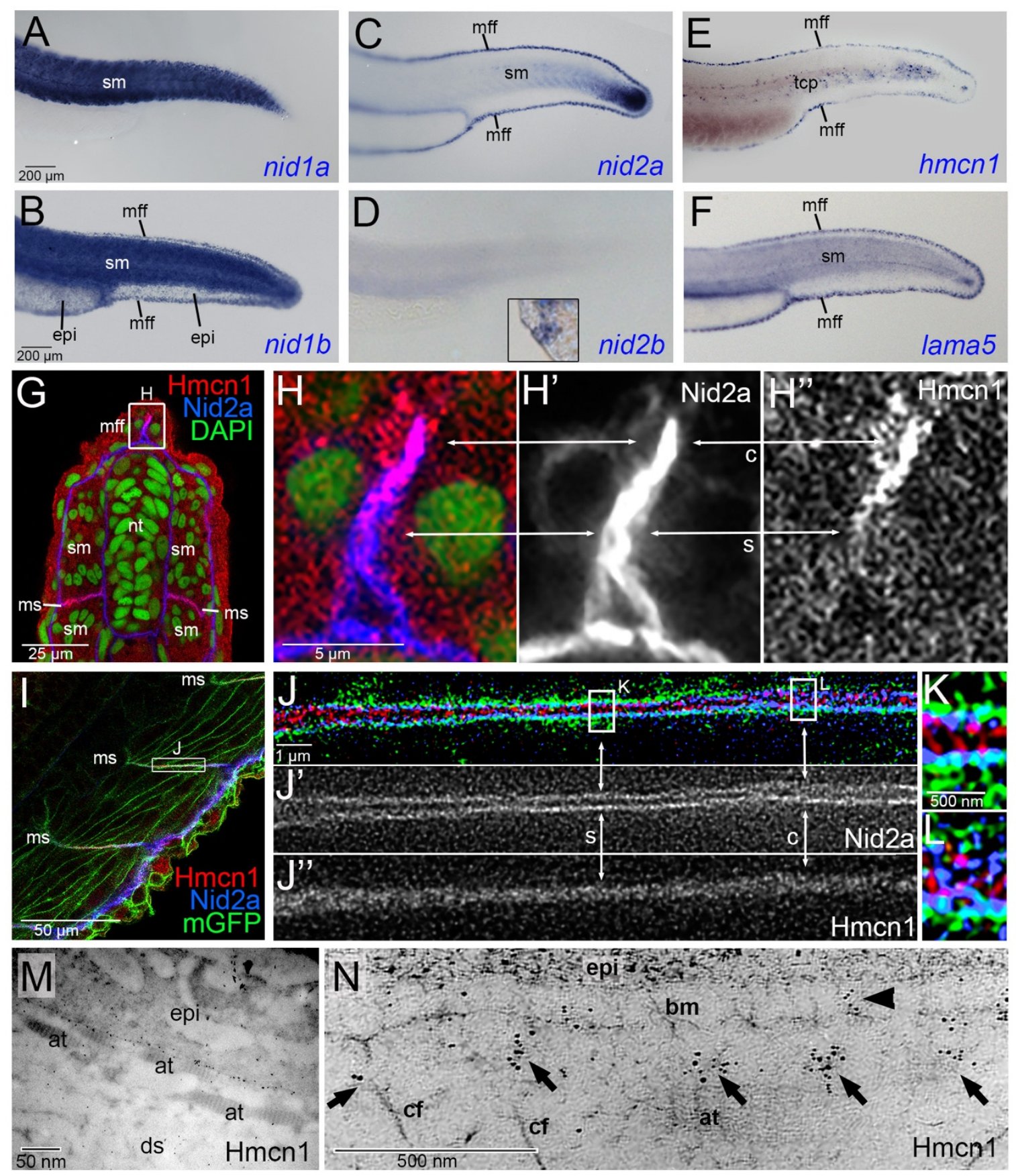

Figure 5. Hmcn1 and Nid2a proteins display partially overlapping localization at basement membranes of zebrafish embryos

(A-F) Lateral views of tails of zebrafish embryos at $30 \mathrm{hpf}$, stained via whole mount in situ hybridization for nid1a, nid1b, nid2a, nid2b, hmcn1 or lama5 transcripts. hmon1 and nid2a as well as lama5 are co-expressed in epidermal cells of the 
median fin fold and in somitic muscle cells. Note the expression of hmcn 1 transcript in tenocyte precursor cells (tcp, E). Inset in D shows labelling of hatching gland cells of same embryo, serving as a positive nid2b control. (G-L) Immunofluorescence analysis of developing dermal-epidermal (G-H') and myotendinous junctions (I-L) of $24 \mathrm{hpf}$ zebrafish, using antibodies directed against Hmcn1 (red) and Nid2 (blue), and counterstained with DAPI (green) to label nuclei $(\mathrm{G}, \mathrm{H})$ or with an antibody against $\operatorname{Tg}(\mathrm{Ola}$.Actb:Hsa.hras-egfp)-encoded GFP to label cell membranes (I-K). $\left(\mathrm{H}-\mathrm{H}^{\prime \prime}\right)$ shows higher magnification of the apical region of dorsal median fin fold boxed in G, (J-J') shows higher magnification of myotendinous junction region boxed in $(I)$, and $(K, L)$ further magnifications of regions boxed in $(J)$. $(G, H, I, J, K, L)$ show merged channels, ( $\left.H^{\prime}, J^{\prime}\right)$ Nid2a single channels and ( $H^{\prime \prime}, J$ ') Hmcn1 single channels. (H-H') Higher magnification of the median fin fold shows Hmcn1 localization largely co-localized with Nid2a in distal, developmentally less advanced regions (50) of the fin fold (arrows labelled with "c"). In comparison, in proximal, developmentally more advanced regions, Hmcn1 and Nid2 localization have started to segregate (arrows labelled with "s"). (J-J") Higher magnification of the mytendinous junction between two myosepta using STED microscopy shows Hmcn1 localization largely separated (indicated by arrows labelled with "s" in J and magnified in $\mathrm{K}$ ) or co-localized (indicated by arrows labelled with "c" in $\mathrm{J}$ and magnified in L) with Nid2a protein. (M) Overview of ultrastructural immunolocalization of Hmcn1 at dermal-epidermal junction of median fin fold of 54 hpf embryo. (N) Higher magnification reveals clusters of Hmcn1 signals distributed underneath (arrows) or even within (arrowhead) the skin BM. Abbreviations: at, actinotrichia; bm, basement membrane; c, co-localized; cf, cross fibers; ds, dermal 
space; epi, epidermis; mGFP, cell membrane-associated GFP; mff, median fin fold;

ms, myoseptum; s, separated; sm, somitic muscle; tcp, tenocyte precursors. 

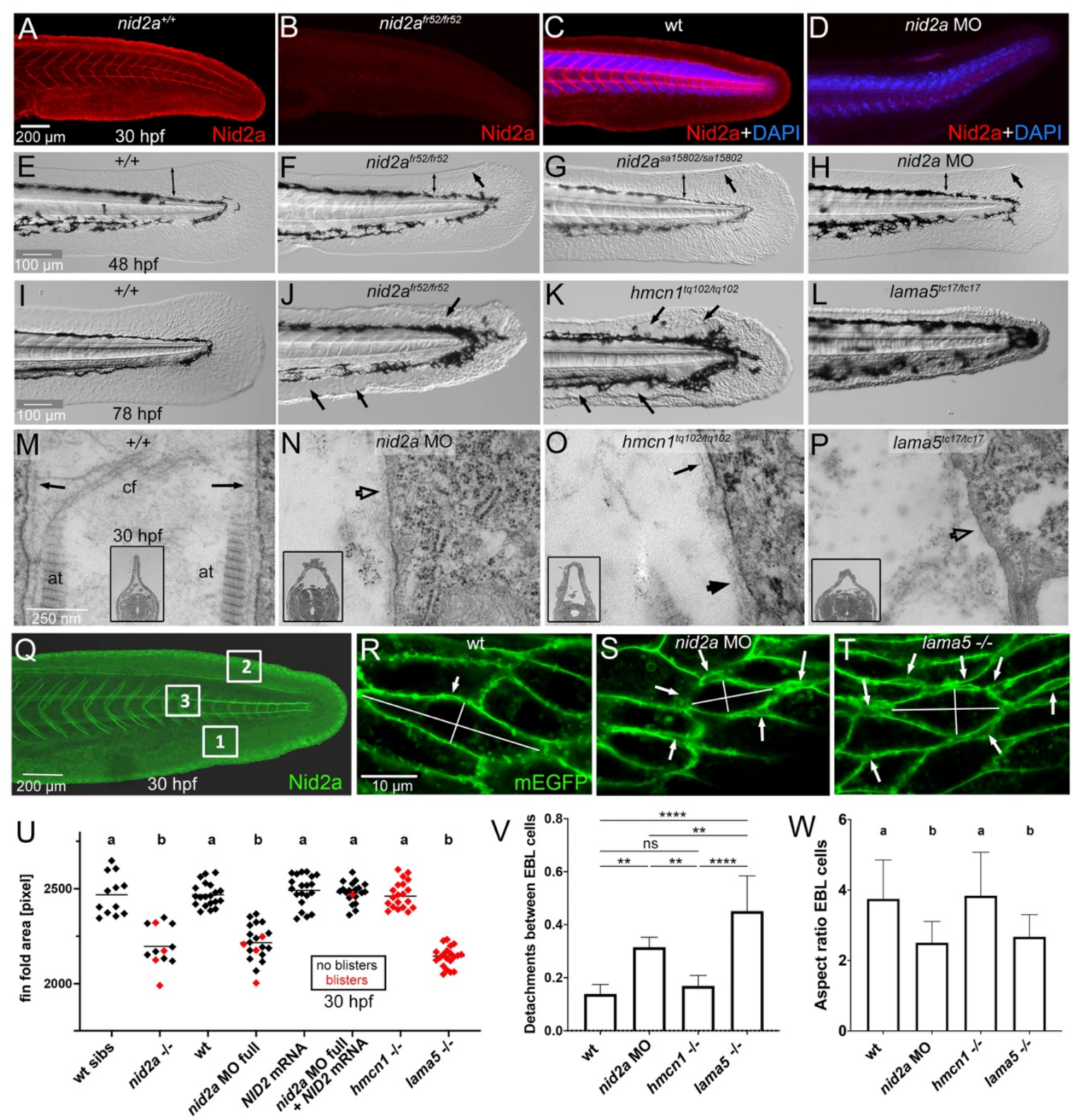

Figure 6. Median fin fold defects of nid2a morphant / mutant zebrafish embryos in comparison to hmen1 and lama5 mutants

(A-D) Immunofluorescence labelling of Nid2a protein, in C,D counterstained with DAPI to label nuclei, of nid2a ${ }^{\text {fr52 }}$ mutant (B), full nid2a morphants (D) and the respective wild-type controls $(A, C), 30 \mathrm{hpf}$, lateral view on tails. For full nid2a knockdowns, also shown in panels $\mathrm{H}, \mathrm{N}, \mathrm{S}, \mathrm{U}-\mathrm{W}, 2.2 \mathrm{ng}$ nid2a-1.ATG MO was injected per embryo (2.5 $\mathrm{nl}$ of $0.1 \mathrm{mM}$ solution). 
$(\mathrm{E}-\mathrm{H})$ Lateral Nomarski images of tails / median fin folds of wild-type, nid2a ${ }^{\text {fr52, }}$ nid2a $a^{\text {sa15802 }}$ and full nid2a morphant, 48 hpf. Bilateral arrows demarcate extension of dorsal median fin folds, regular arrows point to blisters. (I-L) Lateral Nomarski images of tails / median fin folds of wild-type, nid $2 a^{\text {fr52 }}$, hmcn $1^{\text {tq102 }}$ or lama5 $5^{\text {tc17 }}$ mutant embryos, $78 \mathrm{hpf}$. Arrows point to blisters. (M-P) Transmission electron micrographs of dermal-epidermal junctions in dorsal median fin fold of wild-type, nid2a morphant, hmcn1tq102 or lama5 ${ }^{\text {tc17 }}$ mutant embryo, 30 hpf, transverse section through tail region; inset show low magnification overviews of dorsal halves of sections, also illustrating the increased width of the dermal space between the two adjacent epidermal sheets of the dorsal median fin fold in nid2a morphant and hmen1 and lama5 mutants. Arrows point to BM of normal morphology, empty arrow heads in N,P point to regions with indistinct BM in nid2a morphant and lama5 mutant, and filled arrowhead in $\mathrm{O}$ points to regions with widened BM in hmcn1 mutant, consistent with the recently reported BM defects in Hmcn1 mutant mice (49).

(Q) Lateral view on tail region of wild-type embryo, $30 \mathrm{hpf}$, after immunofluorescent labeling of Nid2a (green); boxed regions were used to perform the analyses of keratinocyte detachments and cell shape changes shown in panels R-T,V,W. Regions 1 and 2 are ventral and dorsal median fin fold areas, respectively, and prone to blister formation; region 3 is within body axis and not prone to blistering. (R-S) Representative regions 1 of wild-type, nid2a morphant and lama5 mutant embryo, 30 hpf, double-transgenic for Tg(Ola.Actb:Hsa.hras-egfp) ${ }^{v u 119 T g}$, labeling membranes of all cells with EGFP (green), and $T g(k r t 4: t o m a t o-C A A X)^{\text {fr48Tg, }}$, specifically labeling membranes of outer layer epidermal cells with Tomato to distinguish them from epidermal basal layer (EBL) cells (red channel not shown). 
Arrows point to detachments between basal keratinocytes of the EBL, summarized in panel V, lines indicate the longest and shortest axes of representative cells used to determine the aspect ratios (degree of cellular elongation) summarized in panel W.

(U) Scatter plot of median fin fold sizes, indicating compromised BM-epidermal linkage in 30 hpf nid2a $a^{\text {fr52 }}$, hmcn $1^{\text {tq102 }}$ or lama5 $5^{\text {tc17 }}$ mutant and in 30 hpf wild-type embryos injected with the indicated MOs and/or mRNAs. Each diamond represents one individual embryo. Red diamonds represent embryos with blisters, indicating compromised BM-dermal linkage. Note that co-injection of mouse Nid2 mRNA rescues both the median fin fold size and the blistering defects of nid2a morphant embryos, proving the specificity of the nid2a MO.

$(\mathrm{V}, \mathrm{W})$ Column bar graphs quantifying numbers of detachments between basal keratinocytes of the EBL, normalized against numbers of investigated keratinocytes $(\mathrm{V})$ and the elongation degree (ratio between longest and shortest axis) of such basal keratinocytes of wild-type, nid2a morphant, hmon1 mutants and lama5 mutant embryos, $30 \mathrm{hpf}$, scoring the three tail regions marked in panel $\mathrm{Q}$ and images as shown in panels R-T. Average ratios and standard deviations are indicated.

Significances of differences in U-W were determined via ANOVA followed by the Tukey's post-hoc test. In U,W, groups with different superscript letters $(a, b)$ are significantly different $(p<0.0001)$. In $\mathrm{V},{ }^{* * *}$ and ${ }^{* *}$ indicate significant differences with $p<0.0001$ and $p<0.0070$, respectively. ns indicates no significant differences. Abbreviations: at, actinotrichia; cf, cross fibers; EBL, epidermal basal layer. 
Nid2a + DAPI

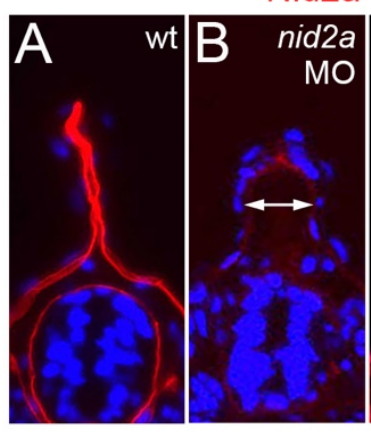

wt

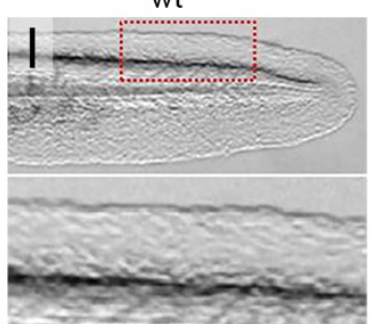

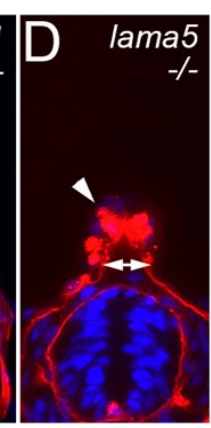

nid2a MO partial

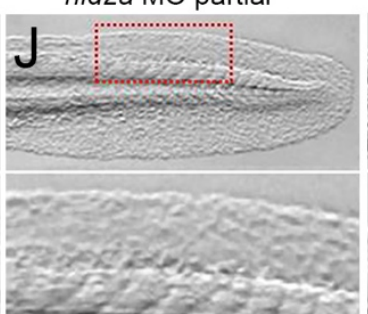

Laminin + DAPI

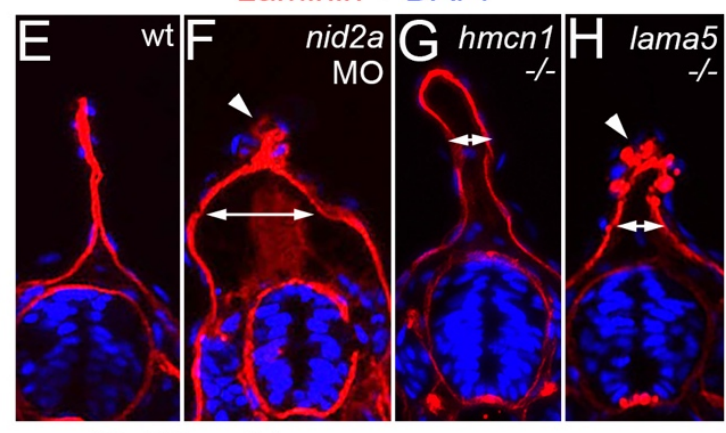

nid2a MO partial hmon1 MOO partial

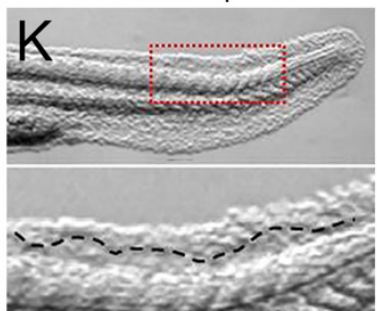

lama5 MO full

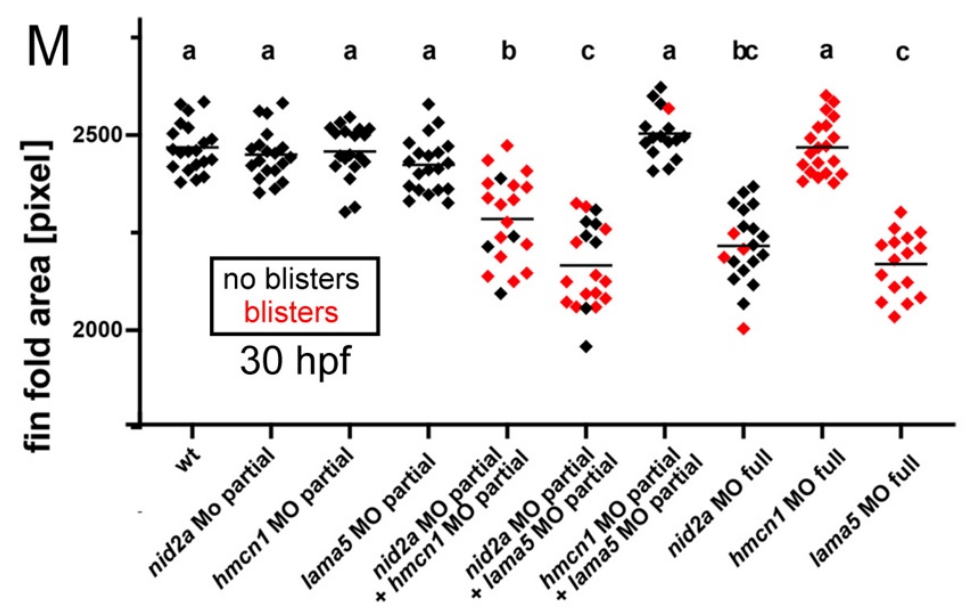

Figure 7. Hmcn1, Nid2a and laminin do not stabilize each other at the protein level but display genetic interaction in vivo.

(A-H) Immunofluorescence labelling of Nid2a (A-D) and Laminin (E-H) (red) of wildtype siblings, nid2a morphants, hmcn1 mutants and lama5 mutants at $30 \mathrm{hpf}$, counterstained with DAPI (blue) to mark nuclei, transverse sections through tail region. Arrows indicate the degree of widening of the dermal space between the two cutaneous BMs of the fin fold labeled by Nid2a or Laminin, indicative of compromised BM-dermal linkage. Arrowheads point to ectopic Nid2a or Laminin 
within the epidermis, most likely resulting from compromised BM and epidermal epithelial integrity. (I-L) Lateral Nomarski images of tails / median fin folds of representative embryos summarized in $\mathrm{M}, 30 \mathrm{hpf}$, after injection with the indicated single or combined MOs. Lower panels show magnified views of region boxed in upper panels. Dashed line in lower panel of $\mathrm{K}$ demarcates region with blister. (M) Quantification of median fin fold sizes (indicative of compromised BM-epidermal linkage) in embryos at $30 \mathrm{hpf}$ after injection with the indicated single or combined MOs. Each diamond represents one individual embryo. Diamonds representing embryos with blisters, indicative of compromised BM-dermal linkage, are in red. Injected amounts of MOs per embryo were: nid2a MO full, 2.2 ng; nid2a MO partial, $1.1 \mathrm{ng} ;$ hmen1 MO full, $4.3 \mathrm{ng}$; hmen1 MO partial, $1.1 \mathrm{ng}$; lama5 MO full, $0.44 \mathrm{ng}$; lama5 MO partial, $0.11 \mathrm{ng}$. For lama5 MO full and hmcn1 MO full, compare with Figure 6 U for lama5 -/- and hmcn1 -/- null mutants. Significances were determined via ANOVA followed by the Tukey's post-hoc test. Groups with different superscript letters $(a, b, c)$ are significantly different ( $a-b$ and $a-c, p<0.0001 ; b-c, p<0.0020)$. 


\section{Supplementary Data}
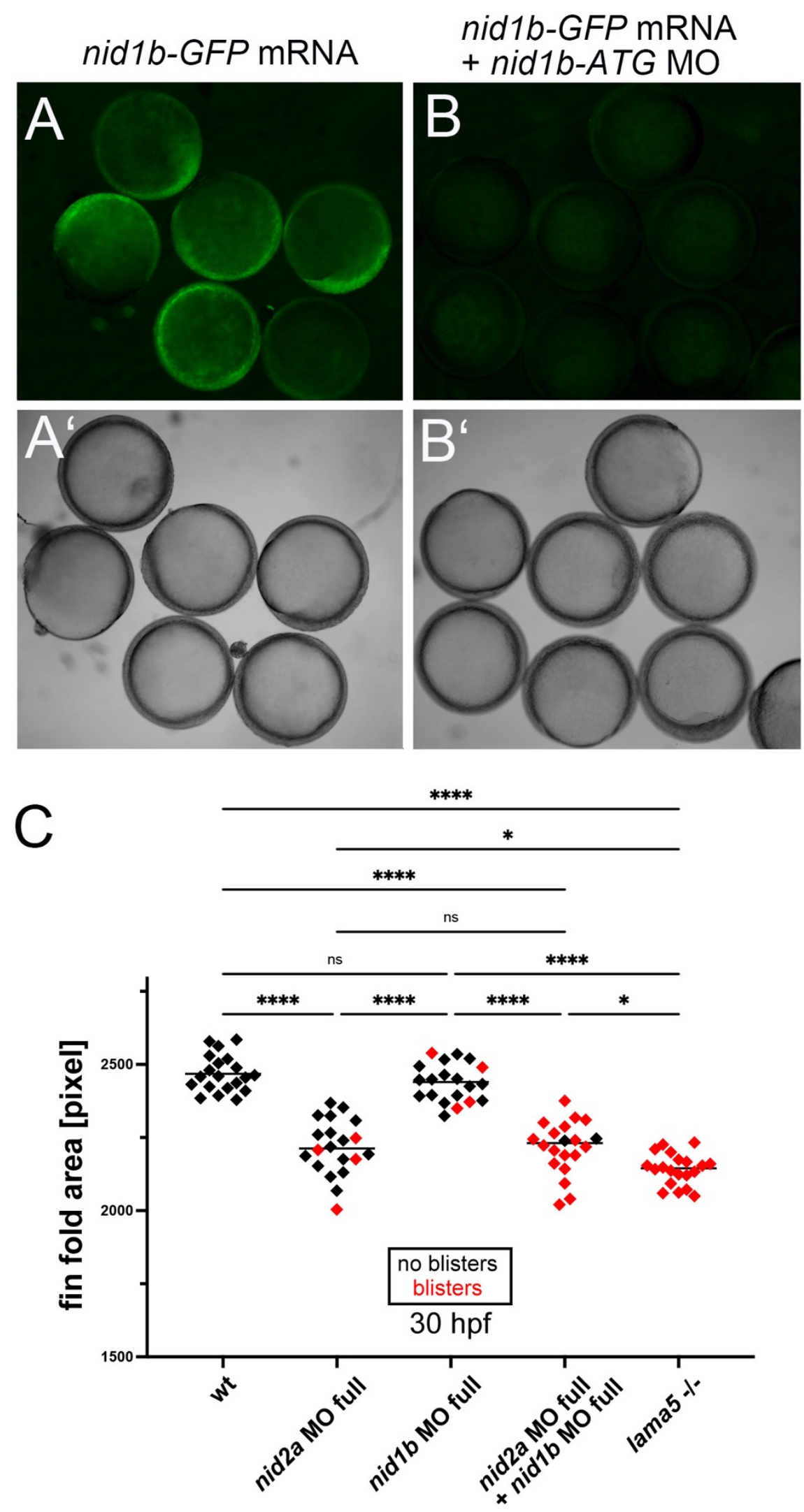
Supplementary Figure S1. Zebrafish nid2a acts in partial functional redundancy with nid1b

$(A, B)$ nid1b-ATG antisense morpholino oligonucleotide efficiently blocks translation of co-injected mRNA encoding a Nid1a-GFP fusion protein. Panels A,B show green fluorescence (to reveal Nid1a-GFP protein) images of embryos injected with nid1bGFP mRNA alone $(\mathrm{A})$ or co-injected with nid1b-GFP mRNA and nid1b-ATG MO (B); panels A',B' the corresponding bright field images. (C) Scatter plot quantifying median fin fold sizes, indicative of compromised BM-epidermal linkage, in $30 \mathrm{hpf}$ wild-type embryos injected with the indicated MOs (2.2 ng per embryo) and, as positive reference, lama5 mutants (compare with Figure 6U). Each diamond represents one individual embryo. Diamonds representing embryos with blisters, indicative of compromised BM-dermal linkage, are in red. In comparison to nid2a single morphants, nid $1 \mathrm{~b} /$ nid $2 \mathrm{a}$ double morphants display increased rates of fin fold blistering. Significances of differences were determined via ANOVA followed by the Tukey's post-hoc test. ${ }^{* * * *}$ indicates a significant difference of $p<0.0001$, and ${ }^{*}$ of $p<0.0178-0.0191$, respectively. ns indicates no significant differences. 